\title{
Synthesis of Benzofurans with Remote Bromide Functionality by Domino 'Ring-Cleavage-Deprotection-Cyclization' Reactions of 2-Alkylidenetetrahydrofurans with Boron Tribromide
}

\author{
Esen Bellur ${ }^{a, b}$ and Peter Langer* a, c \\ ${ }^{a}$ Institut für Chemie, Universität Rostock, \\ Albert-Einstein-Str. 3a, 18051 Rostock, Germany \\ Fax: +381 4986412, E-mail: peter.langer@uni-rostock.de \\ ${ }^{\mathrm{b}}$ Institut für Chemie und Biochemie, Universität Greifswald, \\ Soldmannstr. 16, 17487 Greifswald, Germany \\ ${ }^{\mathrm{c}}$ Leibniz-Institut für Organische Katalyse an der Universität Rostock e. V. (IfOK), \\ Albert-Einstein-Str. 29a, 18059 Rostock, Germany
}

\section{Supplementary Material}

Table of Contents:

$\mathrm{S} 2$

General Comments

$\mathrm{S} 2$

Experimental Section

S20

Copies of NMR spectra 
General Comments. All solvents were dried by standard methods and all reactions were carried out under an inert atmosphere. For the ${ }^{1} \mathrm{H}$ and ${ }^{13} \mathrm{C}$ NMR spectra the deuterated solvents indicated were used. Mass spectral data (MS) were obtained by electron ionization $(70 \mathrm{eV})$, chemical ionization $\left(\mathrm{CI}, \mathrm{H}_{2} \mathrm{O}\right)$ or the electrospray ionization (ESI). For preparative scale chromatography silica gel (60-200 mesh) was used. Melting points are uncorrected.

\section{Experimental Section}

General procedure for the reaction of 2-alkylidenetetrahydrofurans with $\mathrm{N}$ bromosuccinimide (NBS): To a $\mathrm{CCl}_{4}$-solution $(5 \mathrm{~mL} / \mathrm{mmol})$ of the 2alkylidenetetrahydrofuran 2 ( 1 equiv.) was added $N$-bromosuccinimide (1.1 equiv.) at $20{ }^{\circ} \mathrm{C}$. The reaction mixture was heated and stirred at reflux for $3 \mathrm{~h}$. Then, the reaction mixture was allowed to cool down to ambient temperature and the solvent was removed in vacuo. The residue was purified by chromatography (silica gel, $n$-hexane/EtOAc) to give the bromo-2alkylidenetetrahydrofurans 3,4,6.

Synthesis of 3, 4a: Starting with 2a (4.000 g, $28.0 \mathrm{mmol})$ and NBS $(6.511 \mathrm{~g}, 36.6 \mathrm{mmol})$ in $\mathrm{CCl}_{4}(40 \mathrm{~mL}), \mathbf{Z}-3 \mathbf{a}(5.212 \mathrm{~g}, 84 \%)$ and $\boldsymbol{E}$-4a $(0.937 \mathrm{~g}, 11 \%)$ were isolated after chromatography (silica gel, $n$-hexane/EtOAc $=100: 1 \rightarrow 1: 1)$ as yellowish and colorless solids, respectively.

Methyl Bromo(dihydrofuran-2(3H)-ylidene)acetate (Z-3a): ${ }^{1} \mathrm{H} \mathrm{NMR}\left(\mathrm{CDCl}_{3}, 300 \mathrm{MHz}\right): \delta$ 2.23 (quint, $J=7.2 \mathrm{~Hz}, 2 \mathrm{H}, \mathrm{CH}_{2}$ ), 3.19 (t, $J=7.8 \mathrm{~Hz}, 2 \mathrm{H}, \mathrm{CH}_{2}$ ), 3.78 (s, $3 \mathrm{H}, \mathrm{OCH}_{3}$ ), 4.41 (t, 
$\left.J=7.2 \mathrm{~Hz}, 2 \mathrm{H}, \mathrm{OCH}_{2}\right) .{ }^{13} \mathrm{C} \mathrm{NMR}\left(\mathrm{CDCl}_{3}, 75 \mathrm{MHz}\right): \delta 24.8,32.3,52.2,73.0,82.7,164.7$, 172.2. IR (KBr, $\left.\mathrm{cm}^{-1}\right): \widetilde{v}=2951(\mathrm{w}), 1699(\mathrm{~s}), 1609(\mathrm{~s}), 1435(\mathrm{~m}), 1281(\mathrm{~s}), 1213(\mathrm{~s}), 1188$ (m), 1073 (s), 973 (w), 925 (w), 879 (w), 770 (w), 759 (w). MS (EI, 70 eV): m/z (\%) = 221 $\left(\mathrm{M}^{+}\left[{ }^{81} \mathrm{Br}\right], 72\right), 219\left(\mathrm{M}^{+}\left[{ }^{79} \mathrm{Br}\right], 71\right), 190$ (63), 189 (100), 188 (71), 187 (93), 179 (10), 177 (10), 162 (3), 148 (60), 146 (59). Anal. calcd. for $\mathrm{C}_{7} \mathrm{H}_{9} \mathrm{O}_{3} \mathrm{Br}$ (221.050): C, 38.04; H, 4.10; found: C, 38.19; H, 3.64.

Methyl Bromo(3-bromodihydro-furan-2(3H)-ylidene)acetate (E-4a): ${ }^{1} \mathrm{H}$ NMR $\left(\mathrm{CDCl}_{3}\right.$, $300 \mathrm{MHz}): \delta 2.43-2.49\left(\mathrm{~m}, 1 \mathrm{H}, \mathrm{CH}_{2}\right), 2.53-2.61\left(\mathrm{~m}, 1 \mathrm{H}, \mathrm{CH}_{2}\right), 3.80\left(\mathrm{~s}, 3 \mathrm{H}, \mathrm{OCH}_{3}\right), 4.74$ $-4.76\left(\mathrm{~m}, 1 \mathrm{H}, \mathrm{OCH}_{2}\right), 4.78-4.79\left(\mathrm{~m}, 1 \mathrm{H}, \mathrm{OCH}_{2}\right), 5.20(\mathrm{~d}, J=5.4 \mathrm{~Hz}, 1 \mathrm{H}, \mathrm{CH}-\mathrm{Br}) .{ }^{13} \mathrm{C}$ NMR $\left(\mathrm{CDCl}_{3}, 75 \mathrm{MHz}\right): \delta 34.7,48.4,52.1,73.8,87.1,162.0,167.0 . \mathrm{IR}\left(\mathrm{KBr}, \mathrm{cm}^{-1}\right): \widetilde{v}=$ 2950 (w), 1706 (s), 1605 (s), 1433 (m), 1271 (s), 1204 (s), 1174 (s), 1057 (w), 1039 (s), 1027 (s), 1004 (w), $765(\mathrm{w}) . \mathrm{MS}(\mathrm{EI}, 70 \mathrm{eV}): \mathrm{m} / \mathrm{z}(\%)=302\left(\mathrm{M}^{+}\left[2 \times{ }^{81} \mathrm{Br}\right], 17\right), 300\left(\mathrm{M}^{+}\left[{ }^{81} \mathrm{Br}{ }^{79} \mathrm{Br}\right]\right.$, 34), $298\left(\mathrm{M}^{+}\left[2 \times{ }^{79} \mathrm{Br}\right], 17\right), 271(9), 269$ (19), 267 (10), 241 (2), 221 (98), 219 (100), 189 (22), 187 (21), 161 (18), 159 (17), 133 (12), 131 (13), 81 (6), 79 (10). Anal. calcd. for $\mathrm{C}_{7} \mathrm{H}_{8} \mathrm{O}_{3} \mathrm{Br}_{2}$ (299.946): C, 28.03; H, 2.69; found: C, 28.88; H, 2.85.

Synthesis of 3, 4b: Starting with 2b (1.432 g, $9.2 \mathrm{mmol})$ and NBS (2.122 g, $11.92 \mathrm{mmol})$ in $\mathrm{CCl}_{4}(25 \mathrm{~mL}), \mathbf{Z}-4 \mathbf{b}(0.740 \mathrm{~g}, 26 \%), \mathbf{Z}-3 \mathbf{b}(0.886 \mathrm{~g}, 41 \%)$ and $\boldsymbol{E - 4 b}(0.430 \mathrm{~g}, 15 \%)$ were isolated after chromatography (silica gel, $n$-hexane/EtOAc $=100: 1 \rightarrow 1: 1$ ) as a slightly yellowish solid, yellowish solid and as a yellowish oil, respectively.

Ethyl Bromo(3-bromodihydro-furan-2(3H)-ylidene)acetate (Z-4b): ${ }^{1} \mathrm{H}$ NMR $\left(\mathrm{CDCl}_{3}, 300\right.$ MHz): $\delta 1.36\left(\mathrm{t}, J=7.2 \mathrm{~Hz}, 3 \mathrm{H}, \mathrm{CH}_{3}\right), 2.55-2.63\left(\mathrm{~m}, 2 \mathrm{H}, \mathrm{CH}_{2}\right), 4.29(\mathrm{dq}, J=2.1,7.2 \mathrm{~Hz}, 2$ $\left.\mathrm{H}, \mathrm{OCH}_{2} \mathrm{CH}_{3}\right), 4.56-4.66\left(\mathrm{~m}, 2 \mathrm{H}, \mathrm{OCH}_{2}\right), 5.81(\mathrm{dd}, J=4.5,1.5 \mathrm{~Hz}, 1 \mathrm{H}, \mathrm{CH}-\mathrm{Br}) .{ }^{13} \mathrm{C} \mathrm{NMR}$ $\left.\mathrm{CDCl}_{3}, 75 \mathrm{MHz}\right): \delta 13.9,36.8,44.1,61.7,70.6,87.1,162.5,168.5 . \mathrm{IR}\left(\mathrm{KBr}, \mathrm{cm}^{-1}\right): \widetilde{v}=2933$ (w), $1701(\mathrm{~s}), 1616(\mathrm{~s}), 1371(\mathrm{w}), 1274(\mathrm{~s}), 1216(\mathrm{~s}), 1189(\mathrm{~m}), 1160(\mathrm{w}), 1069(\mathrm{~s}), 1026(\mathrm{w})$, 
$953(w), 928$ (w), $872(w), 757(w) . M S(E I, 70 \mathrm{eV}): m / z(\%)=315\left(\mathrm{M}^{+}\left[2 \times{ }^{81} \mathrm{Br}\right], 11\right), 313$ $\left(\mathrm{M}^{+}\left[{ }^{81} \mathrm{Br}{ }^{79} \mathrm{Br}\right], 33\right), 311\left(\mathrm{M}^{+}\left[2 \times{ }^{79} \mathrm{Br}\right], 12\right), 271$ (2), 269 (11), 267 (3), 235 (65), 233 (60), 207 (90), 205 (100), 189 (29), 187 (25), 161 (23), 159 (18), 148 (12), 146 (11), 133 (16), 131 (16). Anal. calcd. for $\mathrm{C}_{8} \mathrm{H}_{10} \mathrm{O}_{3} \mathrm{Br}_{2}$ (313.973): C, 30.60; H, 3.21; found: C, 30.32; H, 3.51.

Ethyl Bromo(dihydrofuran-2(3H)-ylidene)acetate (Z-3b): ${ }^{1} \mathrm{H} \mathrm{NMR}\left(\mathrm{CDCl}_{3}, 300 \mathrm{MHz}\right): \delta$ 1.32 (t, $J=7.2 \mathrm{~Hz}, 3 \mathrm{H}, \mathrm{CH}_{3}$ ), 2.23 (quint, $J=7.5 \mathrm{~Hz}, \mathrm{CH}_{2}$ ), 3.18 (t, $J=7.8 \mathrm{~Hz}, 2 \mathrm{H}, \mathrm{CH}_{2}$ ), $4.23\left(\mathrm{q}, J=7.2 \mathrm{~Hz}, 2 \mathrm{H}, \mathrm{OCH}_{2} \mathrm{CH}_{3}\right), 4.40\left(\mathrm{t}, J=7.2 \mathrm{~Hz}, 2 \mathrm{H}, \mathrm{OCH}_{2}\right) .{ }^{13} \mathrm{C} \mathrm{NMR}\left(\mathrm{CDCl}_{3}, 150\right.$ MHz): $\delta 14.0,24.6,32.2,60.8,72.7,83.0,163.7,171.7 . \mathrm{IR}\left(\mathrm{KBr}, \mathrm{cm}^{-1}\right): \widetilde{v}=2995(\mathrm{w}), 2975$ (w), 2907 (w), 1691 (s), 1608 (s), 1395 (w), 1374 (m), 1294 (s), 1276 (s), 1240 (w), 1194 (s), 1065 (s), 1038 (m), 953 (w), 931 (m), $874(\mathrm{w}), 762(\mathrm{~m}) . \mathrm{MS}(\mathrm{EI}, 70 \mathrm{eV}): \mathrm{m} / \mathrm{z}(\%)=236\left(\mathrm{M}^{+}\right.$ $\left.\left[{ }^{81} \mathrm{Br}\right], 75\right), 234\left(\mathrm{M}^{+}\left[{ }^{79} \mathrm{Br}\right], 76\right), 207$ (44), 205 (43), 190 (100), 188 (93), 164 (11), 162 (11), 148 (45), 146 (45), 110 (40), 81 (12), 79 (9). Anal. calcd. for $\mathrm{C}_{8} \mathrm{H}_{11} \mathrm{O}_{3} \mathrm{Br}$ (235.077): C, 40.88; H, 4.72; found: C, 41.04; H, 4.61 .

E-4b: ${ }^{1} \mathrm{H}$ NMR $\left(\mathrm{CDCl}_{3}, 300 \mathrm{MHz}\right): \delta 1.32\left(\mathrm{t}, J=7.2 \mathrm{~Hz}, 3 \mathrm{H}, \mathrm{CH}_{3}\right), 2.43-2.48(\mathrm{~m}, 1 \mathrm{H}$, $\left.\mathrm{CH}_{2}\right), 2.52-2.61\left(\mathrm{~m}, 1 \mathrm{H}, \mathrm{CH}_{2}\right), 4.27\left(\mathrm{q}, J=7.2 \mathrm{~Hz}, 2 \mathrm{H}, \mathrm{OCH}_{2} \mathrm{CH}_{3}\right), 4.76(\mathrm{dt}, J=8.7,0.9$ $\left.\mathrm{Hz}, 2 \mathrm{H}, \mathrm{OCH}_{2}\right), 5.20(\mathrm{~d}, J=5.4 \mathrm{~Hz}, 1 \mathrm{H}, \mathrm{CH}-\mathrm{Br}) .{ }^{13} \mathrm{C} \mathrm{NMR}\left(\mathrm{CDCl}_{3}, 150 \mathrm{MHz}\right): \delta 14.1$, 35.2, 48.7, 61.5, 74.0, 87.9, 162.0, 176.1. IR (neat, $\left.\mathrm{cm}^{-1}\right): \widetilde{v}=2983(\mathrm{~m}), 2938(\mathrm{w}), 2905(\mathrm{~m})$, 1737 (s), 1703 (s), 1660 (m), 1616 (s), 1471 (m), 1440 (m), 1371 (s), 1316 (m), 1283 (s), 1272 (s), 1206 (s), 1184 (s), 1157 (s), 1117 (m), 1094 (m), 1088 (s), 935 (m), 925 (m), 865 (w), $847(\mathrm{w}), 763(\mathrm{~m}), 700(\mathrm{w}) . \mathrm{MS}(\mathrm{EI}, 70 \mathrm{eV}): \mathrm{m} / \mathrm{z}(\%)=316\left(\mathrm{M}^{+}\left[2 \times{ }^{81} \mathrm{Br}\right], 17\right), 314\left(\mathrm{M}^{+}\right.$ $\left.\left[{ }^{81} \mathrm{Br}{ }^{79} \mathrm{Br}\right], 37\right), 312\left(\mathrm{M}^{+}\left[2 \times{ }^{79} \mathrm{Br}\right], 18\right), 271$ (14), 269 (29), 267 (14), 241 (3), 235 (63), 233 (69), 207 (96), 205 (100), 189 (35), 187 (27), 161 (23), 159 (18), 148 (15), 146 (13), 133 (17), 131 (15), 81 (8), 79 (13). The exact molecular mass $\mathrm{m} / \mathrm{z}=311.8997 \pm 2 \mathrm{ppm}\left[\mathrm{M}^{+}\right]$for $\mathrm{C}_{8} \mathrm{H}_{10} \mathrm{O}_{3} \mathrm{Br}_{2}$ was confirmed by HRMS (EI, $70 \mathrm{eV}$ ). 
Synthesis of 3, 4c: Starting with $2 \mathbf{c}(0.400 \mathrm{~g}, 2.13 \mathrm{mmol})$ and NBS (0.416 g, $2.34 \mathrm{mmol})$ in $\mathrm{CCl}_{4}(30 \mathrm{~mL}), \mathbf{Z}-4 \mathrm{c}(0.087 \mathrm{~g}, 12 \%)$ and $\mathbf{Z - 3 c}(0.302 \mathrm{~g}, 53 \%)$ were isolated after chromatography (silica gel, $n$-hexane/EtOAc $=100: 1 \rightarrow 1: 1$ ) as yellowish oils.

2-Bromo-2-(3-bromodihydrofuran-2(3H)-ylidene)-1-phenylethanone $\quad(Z-4 c):{ }^{1} \mathrm{H} \quad \mathrm{NMR}$ $\left(\mathrm{CDCl}_{3}, 300 \mathrm{MHz}\right): \delta 2.51-2.72\left(\mathrm{~m}, 2 \mathrm{H}, \mathrm{CH}_{2}\right), 4.60-4.71\left(\mathrm{~m}, 2 \mathrm{H}, \mathrm{OCH}_{2}\right), 5.60(\mathrm{~d}, J=5.4$ $\mathrm{Hz}, 1 \mathrm{H}, \mathrm{CH}-\mathrm{Br}), 7.41-7.55(\mathrm{~m}, 3 \mathrm{H}, 3 \times \mathrm{CH}$ of $\mathrm{Ph}), 7.75(\mathrm{~d}, \mathrm{~J}=3.9 \mathrm{~Hz}, 2 \mathrm{H}, 2 \times \mathrm{CH}$ of $\mathrm{Ph})$.

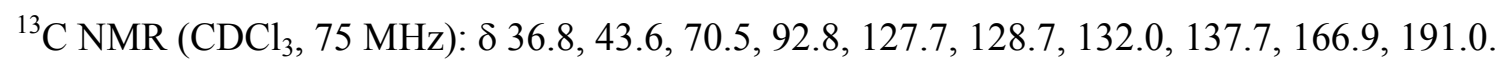
IR (neat, $\mathrm{cm}^{-1}$ ): $\widetilde{v}=3059$ (w), 2998 (w), 2962 (w), 2906 (m), 1659 (s), 1653 (s), 1598 (s), 1584 (s), 1581 (s), 1570 (s), 1473 (w), 1444 (m), 1370 (m), 1311 (s), 1276 (s), 1220 (s), 1174 (s), $1161(\mathrm{~s}), 1115(\mathrm{w}), 1076$ (w), 1055 (m), 1017 (s), 964 (s), 931 (s), 909 (m), 882 (m), 836 (m), $815(\mathrm{w}), 794(\mathrm{~m}), 741(\mathrm{~m}), 687$ (s), $654(\mathrm{~s}), 522(\mathrm{~m}) . \mathrm{MS}(\mathrm{EI}, 70 \mathrm{eV}): \mathrm{m} / \mathrm{z}(\%)=348\left(\mathrm{M}^{+}\right.$ $\left.\left[2 \times{ }^{81} \mathrm{Br}\right], 7\right), 346\left(\mathrm{M}^{+}\left[{ }^{81} \mathrm{Br}{ }^{79} \mathrm{Br}\right], 15\right), 344\left(\mathrm{M}^{+}\left[2 \times{ }^{79} \mathrm{Br}\right], 7\right), 267$ (36), 265 (36), 186 (100), 129 (11), 105 (68), 89 (6), 77 (76). Anal. calcd. for $\mathrm{C}_{12} \mathrm{H}_{10} \mathrm{O}_{2} \mathrm{Br}_{2}$ (346.018): C, 41.65; H, 2.91; found: C, 41.35; H, 3.60.

2-Bromo-2-(dihydrofuran-2(3H)-ylidene)-1-phenylethanone (Z-3c): ${ }^{1} \mathrm{H} \mathrm{NMR}\left(\mathrm{CDCl}_{3}, 300\right.$ $\mathrm{MHz}$ ): $\delta 2.21$ (quint, $J=7.5 \mathrm{~Hz}, 2 \mathrm{H}, \mathrm{CH}_{2}$ ), 2.94 (t, $J=7.8 \mathrm{~Hz}, 2 \mathrm{H}, \mathrm{CH}_{2}$ ), $4.45(\mathrm{t}, J=7.2 \mathrm{~Hz}$, $\left.2 \mathrm{H}, \mathrm{OCH}_{2}\right), 7.38-7.53(\mathrm{~m}, 3 \mathrm{H}, 3 \times \mathrm{CH}$ of $\mathrm{Ph}), 7.61-7.71(\mathrm{~m}, 2 \mathrm{H}, 2 \times \mathrm{CH}$ of $\mathrm{Ph}) .{ }^{13} \mathrm{C} \mathrm{NMR}$ $\left(\mathrm{CDCl}_{3}, 75 \mathrm{MHz}\right): \delta 25.2,32.7,72.9,91.3,127.9$ (2C), 128.5 (2C), 131.4, 139.2, 171.5, 191.8. IR (neat, $\mathrm{cm}^{-1}$ ): $\widetilde{v}=2959$ (w), 2927 (w), 1684 (s), 1646 (s), 1596 (s), 1448 (m), 1420 (w), 1312 (m), 1285 (m), 1253 (w), $1210(\mathrm{~s}), 1183(\mathrm{~m}), 1118(\mathrm{w}), 1075(\mathrm{w}), 1033$ (m), 995 (w), $965(\mathrm{w}), 930(\mathrm{w}), 693(\mathrm{w}), 649(\mathrm{w}) . \mathrm{MS}(\mathrm{EI}, 70 \mathrm{eV}): \mathrm{m} / \mathrm{z}(\%)=267\left(\mathrm{M}^{+}\left[{ }^{81} \mathrm{Br}\right], 22\right), 265$ $\left(\mathrm{M}^{+}\left[{ }^{79} \mathrm{Br}\right], 21\right), 200(11), 198(11), 186(35), 162(3), 148(5), 146(11), 129(2), 105$ (100), 77 (64), 70 (18).

Synthesis of 3d, 6: Starting with 2d (1.000 g, $5.25 \mathrm{mmol})$ and NBS (1.027 g, $5.77 \mathrm{mmol})$ in $\mathrm{CCl}_{4}(50 \mathrm{~mL}), \mathbf{6}(0.680 \mathrm{~g}, 48 \%)$ and $\mathbf{3 d}(0.492 \mathrm{~g}, 35 \%)$ were isolated after chromatography 
( silica gel, $n$-hexane/EtOAc $=100: 1 \rightarrow 2: 1)$ as a yellowish oil and as white solid, respectively.

Methyl (3-Bromo-5-chloromethyldihydrofuran-2(3H)-ylidene)acetate $\quad(6): \quad{ }^{1} \mathrm{H} \quad \mathrm{NMR}$ $\left(\mathrm{CDCl}_{3}, 300 \mathrm{MHz}\right): \delta 2.38-2.71\left(\mathrm{~m}, 2 \mathrm{H}, \mathrm{CH}_{2}\right), 3.73\left(\mathrm{~s}, 3 \mathrm{H}, \mathrm{OCH}_{3}\right), 3.79(\mathrm{dd}, J=9.4,4.9$ $\mathrm{Hz}, 2 \mathrm{H}, \mathrm{CH}_{2}-\mathrm{Cl}$ ), 5.03 (sext, $\left.J=4.9 \mathrm{~Hz}, 1 \mathrm{H}, \mathrm{OCH}\right), 5.33$ (s, $\left.1 \mathrm{H}, \mathrm{CH}=\mathrm{C}\right), 5.81(\mathrm{~d}, J=5.6$ $\mathrm{Hz}, 1 \mathrm{H}, \mathrm{CH}-\mathrm{Br}) .{ }^{13} \mathrm{C} \mathrm{NMR}\left(\mathrm{CDCl}_{3}, 75 \mathrm{MHz}\right): \delta 38.3,43.8,50.5,52.2,79.9,90.7,166.0$, 171.8. IR (neat, $\mathrm{cm}^{-1}$ ): $\widetilde{v}=2953(\mathrm{w}), 1708(\mathrm{~s}), 1653(\mathrm{~s}), 1625(\mathrm{~m}), 1436(\mathrm{~m}), 1407$ (w), 1358 (m), $1335(\mathrm{w}), 1284(\mathrm{~m}), 1243(\mathrm{w}), 1215(\mathrm{~m}), 1194(\mathrm{~m}), 1164(\mathrm{w}), 1125(\mathrm{~s}), 1071(\mathrm{~m}), 1048$ (m), $999(\mathrm{w}), 944(\mathrm{w}), 889(\mathrm{w}), 837(\mathrm{w}), 757(\mathrm{w}), 716(\mathrm{w})$. MS (EI, $70 \mathrm{eV}): \mathrm{m} / \mathrm{z}(\%)=272$ $\left(\mathrm{M}^{+}\left[{ }^{81} \mathrm{Br}{ }^{37} \mathrm{Cl}\right], 21\right), 270\left(\mathrm{M}^{+}\left[{ }^{81} \mathrm{Br}{ }^{35} \mathrm{Cl}\right], 99\right), 268\left(\mathrm{M}^{+}\left[{ }^{79} \mathrm{Br}^{35} \mathrm{Cl}\right], 72\right), 241$ (19), 239 (99), 237 (79), 191 (31), 189 (100), 159 (27), 157 (56), 139 (27), 125 (11), 110 (20), 108 (19), 101 (35), $81(25)$

Methyl Bromo(5-chloromethyldihydrofuran-2(3H)-ylidene)acetate (3d): $\mathrm{mp} .=40{ }^{\circ} \mathrm{C} .{ }^{1} \mathrm{H}$ $\operatorname{NMR}\left(\mathrm{CDCl}_{3}, 300 \mathrm{MHz}\right): \delta 2.11-2.22\left(\mathrm{~m}, 1 \mathrm{H}, \mathrm{CH}_{2}\right), 2.35-2.46\left(\mathrm{~m}, 1 \mathrm{H}, \mathrm{CH}_{2}\right), 3.09-3.21$ (m, $\left.1 \mathrm{H}, \mathrm{CH}_{2}\right), 3.30-3.41\left(\mathrm{~m}, 1 \mathrm{H}, \mathrm{CH}_{2}\right), 3.63-3.73\left(\mathrm{~m}, 2 \mathrm{H}, \mathrm{CH}_{2}-\mathrm{Cl}\right), 3.78\left(\mathrm{~s}, 3 \mathrm{H}, \mathrm{OCH}_{3}\right)$, $4.80-4.87(\mathrm{~m}, 1 \mathrm{H}, \mathrm{OCH}) .{ }^{13} \mathrm{C} \mathrm{NMR}\left(\mathrm{CDCl}_{3}, 75 \mathrm{MHz}\right): \delta 27.3,31.9,45.1,52.1,82.8,82.9$, 164.2, 171.1. IR $\left(\mathrm{KBr}, \mathrm{cm}^{-1}\right): \widetilde{v}=2953(\mathrm{w}), 1701(\mathrm{~s}), 1614(\mathrm{~s}), 1434(\mathrm{~m}), 1346(\mathrm{w}), 1280(\mathrm{~s})$, 1221 (m), 1192 (m), 1122 (w), 1075 (s), 1037 (m), 883 (w), 760 (w), 748 (w), 738 (w). MS $(\mathrm{EI}, 70 \mathrm{eV}): \mathrm{m} / \mathrm{z}(\%)=272\left(\mathrm{M}^{+}\left[{ }^{81} \mathrm{Br}^{37} \mathrm{Cl}\right], 8\right), 270\left(\mathrm{M}^{+}\left[{ }^{81} \mathrm{Br}^{35} \mathrm{Cl}\right], 48\right), 268\left(\mathrm{M}^{+}\left[{ }^{79} \mathrm{Br}^{35} \mathrm{Cl}\right]\right.$, 39), 240 (21), 238 (100), 236 (72), 221 (2), 219 (5), 203 (13), 201 (14), 191 (5), 159 (23), 148 (25), 146 (22), 117 (11), 70 (36). Anal. calcd. for $\mathrm{C}_{8} \mathrm{H}_{10} \mathrm{BrClO}_{3}$ (269.522): C, 35.65; H, 3.74; found: C, 35.38; H, 4.00 .

General procedure for the Suzuki reaction of 2-alkylidenetetrahydrofurans with arylboronic acids: To a 1,4-dioxane solution (3 $\mathrm{mL} / \mathrm{mmol})$ of the bromo-2alkylidenetetrahydrofuran $(\mathbf{3 , 4})$ (1 equiv.) were added potassium phosphate $\left(\mathrm{K}_{3} \mathrm{PO}_{4}, 6\right.$ equiv.), 
the boronic acid $\left(\mathrm{ArB}(\mathrm{OH})_{2}, 3\right.$ equiv. $)$ and $\mathrm{Pd}\left(\mathrm{PPh}_{3}\right)_{4}(0.03$ equiv. $)$ at $20{ }^{\circ} \mathrm{C}$. The reaction mixture was heated and stirred at reflux for $5 \mathrm{~h}$. Then, the reaction mixture was allowed to cool down to ambient temperature and diethyl ether $(10 \mathrm{~mL} / \mathrm{mmol})$ was added. The precipitate was filtered off, washed with diethyl ether and the filtrate was concentrated in vacuo. The residue was purified by chromatography (silica gel, $n$-hexane/EtOAc) to give the aryl-substituted 2-alkylidenetetrahydrofurans 7.

Methyl (Dihydro-furan-2(3H)-ylidene)-(2-methoxy-phenyl)acetate (7a): Starting with 3a (0.100 g, $0.45 \mathrm{mmol}), 2$-methoxyphenylboronic acid (0.213 g, $1.36 \mathrm{mmol}), \mathrm{K}_{3} \mathrm{PO}_{4}(0.575 \mathrm{~g}$, $2.71 \mathrm{mmol})$ and $\mathrm{Pd}\left(\mathrm{PPh}_{3}\right)_{4}(0.016 \mathrm{~g}, 0.014 \mathrm{mmol})$ in 1,4-dioxane $(5 \mathrm{~mL})$, 7a was isolated after chromatography (silica gel, $n$-hexane/EtOAc $=100: 1 \rightarrow 1: 1$ ) as a slightly yellowish solid (0.107 g, 96\%). ${ }^{1} \mathrm{H}$ NMR $\left(\mathrm{CDCl}_{3}, 300 \mathrm{MHz}\right): \delta 2.12$ (quint, $\left.J=7.2 \mathrm{~Hz}, 2 \mathrm{H}, \mathrm{CH}_{2}\right), 3.28(\mathrm{t}, J=$ $\left.7.8 \mathrm{~Hz}, 2 \mathrm{H}, \mathrm{CH}_{2}\right), 3.63\left(\mathrm{~s}, 3 \mathrm{H}, \mathrm{OCH}_{3}\right), 3.77\left(\mathrm{~s}, 3 \mathrm{H}, \mathrm{OCH}_{3}\right), 4.19$ (t, J= 7.2 Hz, $\left.2 \mathrm{H}, \mathrm{OCH}_{2}\right)$, $6.90-6.98(\mathrm{~m}, 2 \mathrm{H}, 2 \times \mathrm{CH}), 7.19(\mathrm{~d}, J=9.3 \mathrm{~Hz}, 1 \mathrm{H}, \mathrm{CH}), 7.24-7.30(\mathrm{~m}, 1 \mathrm{H}, \mathrm{CH}) .{ }^{13} \mathrm{C}$ $\operatorname{NMR}\left(\mathrm{CDCl}_{3}, 75 \mathrm{MHz}\right): \delta 24.0,31.1,51.0,55.5,71.9,100.4,110.9,120.1,124.6,128.3$, 131.8, 157.1, 168.9, 171.5. IR (KBr, $\left.\mathrm{cm}^{-1}\right): \widetilde{v}=2943$ (w), 1704 (s), 1623 (s), 1599 (w), 1492 (m), 1464 (w), 1435 (m), $1376(\mathrm{w}), 1315$ (m), $1271(\mathrm{~m}), 1244$ (m), $1184(\mathrm{~s}), 1114(\mathrm{w}), 1075$ (s), 1068 (s), 1024 (m), 979 (m). MS (EI, 70 eV): m/z (\%) = $248\left(\mathrm{M}^{+}, 100\right), 216(25), 189$ (57), 174 (7), 158 (3). Anal. calcd. for $\mathrm{C}_{14} \mathrm{H}_{16} \mathrm{O}_{4}$ (248.278): C, 67.73; H, 6.50; found: C, $67.51 ; \mathrm{H}, 6.83$.

Methyl (Dihydro-furan-2(3H)-ylidene)-(2,5-dimethoxy-phenyl)acetate (7b): Starting with 3a (0.100 g, $0.45 \mathrm{mmol}), 2,5$-dimethoxybenzeneboronic acid (0.253 g, $1.36 \mathrm{mmol}), \mathrm{K}_{3} \mathrm{PO}_{4}$ $(0.576 \mathrm{~g}, 2.71 \mathrm{mmol})$ and $\mathrm{Pd}\left(\mathrm{PPh}_{3}\right)_{4}(0.016 \mathrm{~g}, 0.014 \mathrm{mmol})$ in 1,4-dioxane $(5 \mathrm{~mL}), 7 \mathbf{b}$ was isolated after chromatography (silica gel, $n$-hexane/EtOAc $=50: 1 \rightarrow 1: 1$ ) as a yellowish solid (0.109 g, 87\%). ${ }^{1} \mathrm{H}$ NMR $\left(\mathrm{CDCl}_{3}, 300 \mathrm{MHz}\right): \delta 2.13$ (quint, $\left.J=7.5 \mathrm{~Hz}, 2 \mathrm{H}, \mathrm{CH}_{2}\right), 3.27$ (t, $J=$ 
$\left.7.8 \mathrm{~Hz}, 2 \mathrm{H}, \mathrm{CH}_{2}\right), 3.64\left(\mathrm{~s}, 3 \mathrm{H}, \mathrm{OCH}_{3}\right), 3.73\left(\mathrm{~s}, 3 \mathrm{H}, \mathrm{OCH}_{3}\right), 3.77\left(\mathrm{~s}, 3 \mathrm{H}, \mathrm{OCH}_{3}\right), 4.20(\mathrm{t}, J=$ $\left.7.2 \mathrm{~Hz}, 2 \mathrm{H}, \mathrm{OCH}_{2}\right), 6.77-6.86(\mathrm{~m}, 3 \mathrm{H}, 3 \times \mathrm{CH}) .{ }^{13} \mathrm{C} \mathrm{NMR}\left(\mathrm{CDCl}_{3}, 150 \mathrm{MHz}\right): \delta 24.2,31.4$, $51.3,55.7,56.5,72.3,100.5,112.2,112.8,118.1,125.9,151.8,153.3,169.0,171.9 . \mathrm{IR}(\mathrm{KBr}$, $\left.\mathrm{cm}^{-1}\right): \widetilde{v}=3009(\mathrm{w}), 2986(\mathrm{w}), 2953(\mathrm{~m}), 2900(\mathrm{w}), 2837(\mathrm{w}), 1700(\mathrm{~s}), 1621(\mathrm{~s}), 1609$ (s), 1497 (s), 1464 (m), 1428 (m), 1377 (w), 1306 (s), 1279 (s), 1236 (s), 1217 (s), 1185 (s), 1160 (m), 1137 (s), 1072 (s), 1053 (s), 1026 (m), 983 (w), 983 (w), 964 (m), 936 (w), 899 (w), 805 (m), 779 (w), $724(\mathrm{w}) . \mathrm{MS}(\mathrm{EI}, 70 \mathrm{eV}): \mathrm{m} / \mathrm{z}(\%)=278\left(\mathrm{M}^{+}, 100\right), 247$ (8), $231(3), 219$ (15), 217 (1), 204 (1), 155 (28), 91 (7), 77 (5), 70 (13). Anal. calcd. for $\mathrm{C}_{15} \mathrm{H}_{18} \mathrm{O}_{5}$ (278.304): C, 64.74; H, 6.52; found: C, 64.75; H, 6.75 .

Ethyl (Dihydro-furan-2(3H)-ylidene)-(2-methoxy-phenyl)acetate (7c): Starting with 3b (0.200 g, $0.85 \mathrm{mmol}), 2$-methoxybenzeneboronic acid (0.400 g, $2.55 \mathrm{mmol}), \mathrm{K}_{3} \mathrm{PO}_{4}(1.085 \mathrm{~g}$, $5.11 \mathrm{mmol})$ and $\mathrm{Pd}\left(\mathrm{PPh}_{3}\right)_{4}(0.030 \mathrm{~g}, 0.026 \mathrm{mmol})$ in 1,4-dioxane $(8 \mathrm{~mL}), 7 \mathrm{c}$ was isolated after chromatography (silica gel, $n$-hexane/EtOAc $=50: 1 \rightarrow 1: 1$ ) as a slightly yellowish solid (0.207 g, 93\%), mp. $=59{ }^{\circ} \mathrm{C} .{ }^{1} \mathrm{H} \mathrm{NMR}\left(\mathrm{CDCl}_{3}, 300 \mathrm{MHz}\right): \delta 1.17\left(\mathrm{t}, J=7.2 \mathrm{~Hz}, 3 \mathrm{H}, \mathrm{CH}_{3}\right)$, 2.12 (quint, $\left.J=7.5 \mathrm{~Hz}, 2 \mathrm{H}, \mathrm{CH}_{2}\right), 3.27$ (t, $\left.J=7.8 \mathrm{~Hz}, 2 \mathrm{H}, \mathrm{CH}_{2}\right), 3.77\left(\mathrm{~s}, 3 \mathrm{H}, \mathrm{OCH}_{3}\right), 4.13$ (q, $\left.J=7.2 \mathrm{~Hz}, 2 \mathrm{H}, \mathrm{OCH}_{2} \mathrm{CH}_{3}\right), 4.18\left(\mathrm{t}, J=7.2 \mathrm{~Hz}, 2 \mathrm{H}, \mathrm{OCH}_{2}\right), 6.88-6.97(\mathrm{~m}, 2 \mathrm{H}, 2 \times \mathrm{CH})$, $7.18-7.29(\mathrm{~m}, 2 \mathrm{H}, 2 \times \mathrm{CH}) .{ }^{13} \mathrm{C} \mathrm{NMR}\left(\mathrm{CDCl}_{3}, 150 \mathrm{MHz}\right): \delta 14.6,24.3,31.4,55.6,59.6$, $72.1,101.1,110.9,120.3,125.0,128.4,132.1,157.4,168.7,171.4 . \operatorname{IR}\left(\mathrm{KBr}, \mathrm{cm}^{-1}\right): \widetilde{v}=29.82$ (m), 2958 (m), 2940 (m), 2900 (m), 2836 (w), 1697 (s), 1627 (s), 1603 (m), 1492 (s), 1462 (m), 1437 (m), 1371 (m), 1304 (s), 1267 (s), 1241 (s), 1185 (s), 1115 (m), 1069 (s), 1028 (m), 1008 (m), 955 (w), 933 (m), 876 (w), 849 (w), 782 (w), 755 (m), 647 (w). MS (EI, 70 eV): $m / z(\%)=262\left(\mathrm{M}^{+}, 100\right), 217(13), 216(15), 189$ (53), 91 (31). Anal. calcd. for $\mathrm{C}_{15} \mathrm{H}_{18} \mathrm{O}_{4}$ (262.305): C, 68.69; H, 6.92; found: C, 68.78; H, 6.81 . 
Ethyl (Dihydro-furan-2(3H)-ylidene)-(2,4-dimethoxy-phenyl)acetate (7d): Starting with 3b (0.150 g, $0.64 \mathrm{mmol}), 2,4-$ dimethoxybenzeneboronic acid (0.355 g, $1.91 \mathrm{mmol}), \mathrm{K}_{3} \mathrm{PO}_{4}$ $(0.813 \mathrm{~g}, 3.83 \mathrm{mmol})$ and $\mathrm{Pd}\left(\mathrm{PPh}_{3}\right)_{4}(0.022 \mathrm{~g}, 0.019 \mathrm{mmol})$ in 1,4-dioxane $(10 \mathrm{~mL}), 7 \mathbf{d}$ was isolated after chromatography (silica gel, $n$-hexane/EtOAc $=50: 1 \rightarrow 1: 1$ ) as a white solid (0.180 g, 96\%), mp. $=91{ }^{\circ} \mathrm{C} .{ }^{1} \mathrm{H} \mathrm{NMR}\left(\mathrm{CDCl}_{3}, 300 \mathrm{MHz}\right): \delta 1.17\left(\mathrm{t}, J=7.2 \mathrm{~Hz}, 3 \mathrm{H}, \mathrm{CH}_{3}\right)$, 2.11 (quint, $J=7.5 \mathrm{~Hz}, 2 \mathrm{H}, \mathrm{CH}_{2}$ ), $3.25\left(\mathrm{t}, J=7.8 \mathrm{~Hz}, 2 \mathrm{H}, \mathrm{CH}_{2}\right), 3.75\left(\mathrm{~s}, 3 \mathrm{H}, \mathrm{OCH}_{3}\right), 3.81$ $\left(\mathrm{s}, 3 \mathrm{H}, \mathrm{OCH}_{3}\right), 4.12\left(\mathrm{q}, J=7.2 \mathrm{~Hz}, 2 \mathrm{H}, \mathrm{OCH}_{2} \mathrm{CH}_{3}\right), 4.18\left(\mathrm{t}, J=7.2 \mathrm{~Hz}, 2 \mathrm{H}, \mathrm{OCH}_{2}\right), 6.48-$ $6.51(\mathrm{~m}, 2 \mathrm{H}, 2 \times \mathrm{CH}), 7.08-7.11(\mathrm{~m}, 1 \mathrm{H}, \mathrm{CH}) .{ }^{13} \mathrm{C} \mathrm{NMR}\left(\mathrm{CDCl}_{3}, 150 \mathrm{MHz}\right): \delta 14.4,24.1$, $31.1,55.1,55.3,59.4,71.8,98.5,100.5,103.9,117.4,132.1,158.1,159.9,168.6,171.09$. IR $\left(\mathrm{KBr}, \mathrm{cm}^{-1}\right): \widetilde{v}=3000(\mathrm{w}), 2977(\mathrm{w}), 1690(\mathrm{~s}), 1616$, (s), $1584(\mathrm{w}), 1511(\mathrm{~m}), 1464(\mathrm{w})$, 1309 (m), 1289 (m), 1265 (m), 1209 (m), 1187 (m), 1167 (m), 1108 (w), 1069 (s), 1032 (m), $1008(w), 947(w), 819(w)$. MS (EI, 70 eV): m/z (\%) = $292\left(\mathrm{M}^{+}, 100\right), 246(20), 231(1), 219$ (16), 190 (8). Anal. calcd. for $\mathrm{C}_{16} \mathrm{H}_{20} \mathrm{O}_{5}$ (292.331): C, 65.74; H, 6.90; found: C, 65.91; H, 6.87.

Ethyl (Dihydro-furan-2(3H)-ylidene)-(2,6-dimethoxy-phenyl)acetate (7e): Starting with 3b $(0.170 \mathrm{~g}, 0.72 \mathrm{mmol}), 2,6$-dimethoxybenzeneboronic acid (0.403 g, $2.17 \mathrm{mmol}), \mathrm{K}_{3} \mathrm{PO}_{4}$ $(0.921 \mathrm{~g}, 4.34 \mathrm{mmol})$ and $\mathrm{Pd}\left(\mathrm{PPh}_{3}\right)_{4}(0.025 \mathrm{~g}, 0.022 \mathrm{mmol})$ in 1,4-dioxane $(10 \mathrm{~mL}), 7 \mathrm{e}$ was isolated after chromatography (silica gel, $n$-hexane/EtOAc $=100: 1 \rightarrow 1: 1$ ) as a yellowish solid (0.182 g, 87\%), mp. $=82{ }^{\circ} \mathrm{C} .{ }^{1} \mathrm{H} \mathrm{NMR}\left(\mathrm{CDCl}_{3}, 300 \mathrm{MHz}\right): \delta 1.15$ (t, J=7.2 Hz, $3 \mathrm{H}$, $\mathrm{CH}_{3}$ ), 2.11 (quint, $\left.J=7.2 \mathrm{~Hz}, 2 \mathrm{H}, \mathrm{CH}_{2}\right), 3.29\left(\mathrm{t}, J=7.5 \mathrm{~Hz}, 2 \mathrm{H}, \mathrm{CH}_{2}\right), 3.76(\mathrm{~s}, 6 \mathrm{H}$, $\left.2 \times \mathrm{OCH}_{3}\right), 4.11\left(\mathrm{q}, J=7.2 \mathrm{~Hz}, 2 \mathrm{H}, \mathrm{OCH}_{2} \mathrm{CH}_{3}\right), 4.16\left(\mathrm{t}, J=7.2 \mathrm{~Hz}, 2 \mathrm{H}, \mathrm{OCH}_{2}\right), 6.59(\mathrm{~d}, J=$ $8.4 \mathrm{~Hz}, 2 \mathrm{H}, 2 \times \mathrm{CH}), 7.22(\mathrm{t}, J=8.4 \mathrm{~Hz}, 1 \mathrm{H}, \mathrm{CH}) .{ }^{13} \mathrm{C} \mathrm{NMR}\left(\mathrm{CDCl}_{3}, 150 \mathrm{MHz}\right): \delta 14.7,24.3$, $31.3,56.2,59.5,72.0,96.3,104.4,113.8,128.7,158.4,168.8,171.8$. IR (neat, $\left.\mathrm{cm}^{-1}\right): \widetilde{v}=$ 2976 (m), 2926 (m), 2855 (w), 1696 (s), 1630 (s), 1589 (m), 1468 (s), 1436 (m), 1378 (w), 1286 (m), 1248 (s), 1182 (s), 1112 (s), 1067 (s), 1030 (m), 931 (w), 746 (w). MS (EI, 70 eV): 
$m / z(\%)=292\left(\mathrm{M}^{+}, 95\right), 263(6), 219(100), 204(4), 190(15), 188(4), 173(8), 157$ (3), 137 (5). Anal. calcd. for $\mathrm{C}_{16} \mathrm{H}_{20} \mathrm{O}_{5}$ (292.331): C, 65.74; H, 6.90; found: $\mathrm{C}, 65.61 ; \mathrm{H}, 6.24$.

2-(Dihydro-furan-2(3H)-ylidene)-2-(2-methoxy-phenyl)-1-phenyl-ethanone (7f): Starting with 3c (0.100 g, $0.374 \mathrm{mmol})$, 2-methoxyphenylboronic acid (0.176 g, $1.12 \mathrm{mmol}), \mathrm{K}_{3} \mathrm{PO}_{4}$ $(0.478 \mathrm{~g}, 2.25 \mathrm{mmol})$ and $\mathrm{Pd}\left(\mathrm{PPh}_{3}\right)_{4}(0.013 \mathrm{~g}, 0.011 \mathrm{mmol})$ in 1,4-dioxane $(10 \mathrm{~mL}), 7 \mathbf{f}$ was isolated after chromatography ( solid $(0.085 \mathrm{~g}, 77 \%), \mathrm{mp} .=123{ }^{\circ} \mathrm{C} .{ }^{1} \mathrm{H} \mathrm{NMR}\left(\mathrm{CDCl}_{3}, 300 \mathrm{MHz}\right): \delta 2.16$ (quint, $J=7.5 \mathrm{~Hz}, 2$ $\left.\mathrm{H}, \mathrm{CH}_{2}\right), 3.22\left(\mathrm{t}, J=7.8 \mathrm{~Hz}, 2 \mathrm{H}, \mathrm{CH}_{2}\right), 3.44\left(\mathrm{~s}, 3 \mathrm{H}, \mathrm{OCH}_{3}\right), 4.27$ (t, $\left.J=7.2 \mathrm{~Hz}, 2 \mathrm{H}, \mathrm{OCH}_{2}\right)$, $6.63(\mathrm{dd}, J=5.1,1.2 \mathrm{~Hz}, 1 \mathrm{H}, \mathrm{CH}), 6.94(\mathrm{dt}, J=7.5,1.2 \mathrm{~Hz}, 1 \mathrm{H}, \mathrm{CH}), 7.11-7.18(\mathrm{~m}, 3 \mathrm{H}$, $3 \times \mathrm{CH}$ of $\mathrm{Ar}), 7.22-7.28(\mathrm{~m}, 1 \mathrm{H}, \mathrm{CH}), 7.45(\mathrm{dt}, J=7.5,1.5 \mathrm{~Hz}, 3 \mathrm{H}, 3 \times \mathrm{CH}$ of $\mathrm{Ar}) .{ }^{13} \mathrm{C} \mathrm{NMR}$ $\left(\mathrm{CDCl}_{3}, 50 \mathrm{MHz}\right): \delta 24.2,31.4,54.8,71.8,108.5,110.7,120.2,126.3,127.0(2 \mathrm{C}), 128.1$ (2C), 128.2, 130.1, 131.8, 140.6, 156.3, 171.3, 196.1. IR $\left(\mathrm{KBr}, \mathrm{cm}^{-1}\right): \widetilde{v}=2992(\mathrm{w}), 2987$ (w), 2936 (w), 1654 (s), 1595 (s), 1581 (s), 1487 m), 1458 (m), 1443 (w), $1322(\mathrm{~m}), 1300(\mathrm{w})$, 1260 (s), 1249 (s), 1186 (s), 1114 (m), 1051 (w), 1022 (m), 967 (s), 932 (m), 889 (m), 760 (m), $712(\mathrm{w}), 712(\mathrm{w}), 663(\mathrm{w}) . \mathrm{MS}(\mathrm{EI}, 70 \mathrm{eV}): \mathrm{m} / \mathrm{z}(\%)=294\left(\mathrm{M}^{+}, 88\right), 263$ (29), 217 (39, 189 (28), 171 (4), 157 (1), 105 (100), 91 (17), 77 (62).

\section{Methyl (5-Chloromethyl-dihydro-furan-2(3H)-ylidene)-(2-methoxy-phenyl)acetate (7g):}

Starting with 3d (0.150 g, $0.56 \mathrm{mmol}), 2$-methoxyphenylboronic acid (0.262 g, $1.67 \mathrm{mmol})$, $\mathrm{K}_{3} \mathrm{PO}_{4}(0.709 \mathrm{~g}, 3.34 \mathrm{mmol})$ and $\left[\mathrm{Pd}\left(\mathrm{PPh}_{3}\right)_{4}\right](0.019 \mathrm{~g}, 0.017 \mathrm{mmol})$ in 1,4-dioxane $(5 \mathrm{~mL})$, $7 \mathbf{g}$ was isolated after chromatography (silica gel, $n$-hexane/EtOAc $=100: 1 \rightarrow 1: 1$ ) as a slightly yellowish oil (0.159 g, 96\%). ${ }^{1} \mathrm{H}$ NMR $\left(\mathrm{CDCl}_{3}, 300 \mathrm{MHz}\right): \delta 2.02-2.14(\mathrm{~m}, 1 \mathrm{H}$, $\left.\mathrm{CH}_{2}\right), 2.19-2.31\left(\mathrm{~m}, 1 \mathrm{H}, \mathrm{CH}_{2}\right), 3.26-3.36\left(\mathrm{~m}, 2 \mathrm{H}, \mathrm{CH}_{2}\right), 3.54\left(\mathrm{~d}, J=5.1 \mathrm{~Hz}, 2 \mathrm{H}, \mathrm{CH}_{2}-\right.$ $\mathrm{Cl}), 3.60\left(\mathrm{~s}, 3 \mathrm{H}, \mathrm{OCH}_{3}\right), 3.75\left(\mathrm{~s}, 3 \mathrm{H}, \mathrm{OCH}_{3}\right), 4.58$ (quint, $\left.J=7.2 \mathrm{~Hz}, 1 \mathrm{H}, \mathrm{OCH}\right), 6.87-6.96$ (m, $2 \mathrm{H}, 2 \times \mathrm{CH}$ of $\mathrm{Ar}), 7.18-7.27(\mathrm{~m}, 2 \mathrm{H}, 2 \times \mathrm{CH}$ of $\mathrm{Ar}) .{ }^{13} \mathrm{C} \mathrm{NMR}\left(\mathrm{CDCl}_{3}, 75 \mathrm{MHz}\right): \delta 26.6$, 
$30.7,45.4,51.0,53.3,81.6,100.9,110.6,120.0,124.2,128.3,131.8,157.0,168.7,170.3$. IR (neat, $\mathrm{cm}^{-1}$ ): $\widetilde{v}=2950(\mathrm{w}), 17004$ (s), 1632 (s), $1492(\mathrm{~m}), 1460$ (m), 1435 (s), $1346(\mathrm{w})$, 1308 (m), 1265 (m), 1244 (s), 1185 (s), 1116 (m), 1072 (s), 1026 (m), 926 (w), 755 (m). MS $(\mathrm{EI}, 70 \mathrm{eV}): \mathrm{m} / \mathrm{z}(\%)=298\left(\mathrm{M}^{+}\left[{ }^{37} \mathrm{Cl}\right], 33\right), 296\left(\mathrm{M}^{+}\left[{ }^{35} \mathrm{Cl}\right], 100\right), 266(17), 264$ (50), 239 (16), 237 (58), 201 (7), $176(10), 174$ (13), 150 (13), 148 (39).

\section{Methyl (2-Methoxy-phenyl)-[3-(2-methoxy-phenyl)-dihydro-furan-2(3H)-ylidene]acetate}

(7h): Starting with Z-4a (0.300 g, $1.0 \mathrm{mmol})$, 2-methoxybenzeneboronic acid (0.940 g, 6.0 $\mathrm{mmol}), \mathrm{K}_{3} \mathrm{PO}_{4}(1.274 \mathrm{~g}, 6.0 \mathrm{mmol})$ and $\left[\mathrm{Pd}\left(\mathrm{PPh}_{3}\right)_{4}\right](0.058 \mathrm{~g}, 0.05 \mathrm{mmol})$ in 1,4-dioxane (5 $\mathrm{mL}$ ), $7 \mathbf{h}$ was isolated after chromatography (silica gel, $n$-hexane/EtOAc $=100: 1 \rightarrow 2: 1$ ) as a white solid $(0.325 \mathrm{~g}, 92 \%), \mathrm{mp} .=112{ }^{\circ} \mathrm{C} .{ }^{1} \mathrm{H}$ NMR $\left(\mathrm{CDCl}_{3}, 300 \mathrm{MHz}\right): \delta 1.58-2.05(\mathrm{~m}, 1$ $\left.\mathrm{H}, \mathrm{CH}_{2}\right), 2.41-2.56(\mathrm{~m}, 1 \mathrm{H}, \mathrm{CH}), 3.43\left(\mathrm{~s}, 3 \mathrm{H}, \mathrm{OCH}_{3}\right), 3.82\left(\mathrm{~s}, 3 \mathrm{H}, \mathrm{OCH}_{3}\right), 3.91(\mathrm{~s}, 3 \mathrm{H}$, $\left.\mathrm{OCH}_{3}\right), 4.04-4.18\left(\mathrm{~m}, 1 \mathrm{H}, \mathrm{OCH}_{2}\right), 4.24\left(\mathrm{dt}, J=8.4,1.8 \mathrm{~Hz}, 1 \mathrm{H}, \mathrm{OCH}_{2}\right), 5.34(\mathrm{dd}, J=8.7$, $1.5 \mathrm{~Hz}, 1 \mathrm{H}, \mathrm{CH}), 6.86-7.05(\mathrm{~m}, 4 \mathrm{H}, 4 \times \mathrm{CH}), 7.19-7.32(\mathrm{~m}, 2 \mathrm{H}, 2 \times \mathrm{CH}), 7.37-7.45(\mathrm{~m}, 1$ $\mathrm{H}, \mathrm{CH}), 7.81-7.85(\mathrm{~m}, 1 \mathrm{H}, \mathrm{CH}) .{ }^{13} \mathrm{C} \mathrm{NMR}\left(\mathrm{CDCl}_{3}, 75 \mathrm{MHz}\right): \delta 32.7,42.7,50.9,55.4,55.6$, $70.0,101.9,110.4,111.0,120.1,120.2,125.0,127.0,127.6,128.4,130.0,131.8,156.5,157.3$, 167.7, 172.7. IR $\left(\mathrm{KBr}, \mathrm{cm}^{-1}\right): \widetilde{v}=2946(\mathrm{w}), 1705(\mathrm{~m}), 1631(\mathrm{~m}), 1600(\mathrm{~s}), 1577(\mathrm{~m}), 1489$ (s), 1461 (s), 1436 (s), 1402 (m), 1368 (s), 1344 (s), 1296 (m), 1270 (m), 1234 (s), 1197 (w), 1166 (m), 1109 (m), 1076 (s), 1054 (s), 1024 (s), 982 (w), 779 (m), 758 (s), 704 (w), 656 (m), $528(\mathrm{w}) . \mathrm{MS}(\mathrm{EI}, 70 \mathrm{eV}): \mathrm{m} / \mathrm{z}(\%)=354\left(\mathrm{M}^{+}, 100\right), 322$ (39), 295 (25), 247 (32). The exact molecular mass $\mathrm{m} / \mathrm{z}=354.1467 \pm 2 \mathrm{ppm}\left[\mathrm{M}^{+}\right]$for $\mathrm{C}_{21} \mathrm{H}_{22} \mathrm{O}_{5}$ was confirmed by HRMS (EI, 70 $\mathrm{eV})$.

\section{General procedure for the synthesis of benzofurans by reaction of 2-} alkylidenetetrahydrofurans with borontribromide: To a $\mathrm{CH}_{2} \mathrm{Cl}_{2}$-solution $(10 \mathrm{~mL} / \mathrm{mmol})$ of the 2-alkylidenetetrahydrofuran 7 (1 equiv.) was added $\mathrm{BBr}_{3}\left(4\right.$ equiv.) at $0{ }^{\circ} \mathrm{C}$. The reaction 
mixture was allowed to warm to $20{ }^{\circ} \mathrm{C}$ over $12 \mathrm{~h}$ and was stirred for $6 \mathrm{~h}$ at $20{ }^{\circ} \mathrm{C}$. Water $(15$ $\mathrm{mL} / \mathrm{mmol}$ substrate) was slowly added to the reaction mixture and the organic layer was separated. The aqueous layer was extracted with $\mathrm{CH}_{2} \mathrm{Cl}_{2}(3 \times 30 \mathrm{~mL} / \mathrm{mmol}$ substrate $)$. The combined organic extracts were dried $\left(\mathrm{Na}_{2} \mathrm{SO}_{4}\right)$, filtered and the filtrate was concentrated in vacuo. The residue was purified by chromatography (silica gel, $n$-hexane/EtOAc) to give $\mathbf{8}$.

Methyl 2-(3'-Bromopropyl)benzofuran-3-carboxylate (8a): Starting with 7a $(0.150 \mathrm{~g}, 0.60$ mmol) and $\mathrm{BBr}_{3}(0.605 \mathrm{~g}, 2.42 \mathrm{mmol})$ in $\mathrm{CH}_{2} \mathrm{Cl}_{2}(6 \mathrm{~mL})$, 8a was isolated after chromatography (silica gel, $n$-hexane/EtOAc $=100: 1 \rightarrow 1: 1)$ as a yellowish oil $(0.163 \mathrm{~g}$, 92\%). ${ }^{1} \mathrm{H} \mathrm{NMR}\left(\mathrm{CDCl}_{3}, 300 \mathrm{MHz}\right): \delta 2.34$ (quint, $\left.J=7.2 \mathrm{~Hz}, 2 \mathrm{H}, \mathrm{CH}_{2}\right), 3.35(\mathrm{t}, J=7.5 \mathrm{~Hz}, 2$ $\left.\mathrm{H}, \mathrm{CH}_{2}\right), 3.47$ (t, J=6.9 Hz, $\left.2 \mathrm{H}, \mathrm{CH}_{2}-\mathrm{Br}\right), 3.95\left(\mathrm{~s}, 3 \mathrm{H}, \mathrm{OCH}_{3}\right), 7.28-7.33(\mathrm{~m}, 2 \mathrm{H}, 2 \times \mathrm{CH})$, $7.42-7.45(\mathrm{~m}, 1 \mathrm{H}, \mathrm{CH}), 7.95-7.98(\mathrm{~m}, 1 \mathrm{H}, \mathrm{CH}) .{ }^{13} \mathrm{C} \mathrm{NMR}\left(\mathrm{CDCl}_{3}, 75 \mathrm{MHz}\right): \delta 26.8$, $30.8,32.4,51.4,109.2,110.9,121.9,123.9,124.6,125.8,153.6,164.5,165.2$. IR (neat, $\mathrm{cm}^{-1}$ ): $\widetilde{v}=2952(\mathrm{~m}), 1714(\mathrm{~s}), 1593(\mathrm{~s}), 1478(\mathrm{~m}), 1451(\mathrm{~s}), 1437(\mathrm{~s}), 1386(\mathrm{~m}), 1342(\mathrm{w}), 1284$ (m), 1235 (s), 1174 (s), 1127 (w), 1106 (m), 1073 (s), 1010 (w), 959 (w), 935 (w), 861 (w), 790 (m), 752 (s). MS (EI, $70 \mathrm{eV}): \mathrm{m} / \mathrm{z}(\%)=298\left(\mathrm{M}^{+}\left[{ }^{81} \mathrm{Br}\right], 38\right), 296\left(\mathrm{M}^{+}\left[{ }^{79} \mathrm{Br}\right], 39\right), 267$ (7), 265 (7), 217 (16), 203 (10), 201 (6), 189 (100), 175 (5), 171 (29), 159 (47), 157 (22), 145 (4), 131 (34), 114 (12), 102 (37), 77 (14). Anal. calcd. for $\mathrm{C}_{13} \mathrm{H}_{13} \mathrm{BrO}_{3}$ (297.148): C, 52.55; H, 4.41; found: C, 52.84; H, 4.74.

Methyl 2-(3'-Bromopropyl)-5-hydroxybenzofuran-3-carboxylate (8b): Starting with 7b $(0.070 \mathrm{~g}, 0.25 \mathrm{mmol})$ and $\mathrm{BBr}_{3}(0.504 \mathrm{~g}, 2.0 \mathrm{mmol})$ in $\mathrm{CH}_{2} \mathrm{Cl}_{2}(5 \mathrm{~mL}), \mathbf{8 b}$ was isolated after chromatography (silica gel, $n$-hexane/EtOAc $=100: 1 \rightarrow 5: 1)$ as a white solid $(0.076 \mathrm{~g}, 97 \%)$, mp. $=154{ }^{\circ} \mathrm{C} .{ }^{1} \mathrm{H} \mathrm{NMR}\left(\mathrm{CDCl}_{3}, 300 \mathrm{MHz}\right): \delta 2.34$ (quint, $\left.J=7.05 \mathrm{~Hz}, 2 \mathrm{H}, \mathrm{CH}_{2}\right), 3.33(\mathrm{t}, J=$ $7.5 \mathrm{~Hz}, 2 \mathrm{H}, \mathrm{CH}_{2}$ ), 3.47 (t, J=6.6 Hz, $2 \mathrm{H}, \mathrm{CH}_{2}-\mathrm{Br}$ ), 3.95 (s, $3 \mathrm{H}, \mathrm{OCH}_{3}$ ), 5.31 (broad s, $1 \mathrm{H}$, OH), $6.83(\mathrm{dd}, J=8.7,2.4 \mathrm{~Hz}, 1 \mathrm{H}, \mathrm{CH}), 7.30(\mathrm{~d}, J=8.7 \mathrm{~Hz}, 1 \mathrm{H}, \mathrm{CH}), 7.46(\mathrm{~d}, J=2.4 \mathrm{~Hz}, 1$ 
$\mathrm{H}, \mathrm{CH}) .{ }^{13} \mathrm{C}$ NMR $\left(\mathrm{CDCl}_{3} / \mathrm{DMSO}_{6}, 150 \mathrm{MHz}\right): \delta 26.4,30.2,32.2,50.8,106.3,108.4$, 110.6, 112.9, 126.1, 147.4, 153.7, 163.9, 165.0. IR $\left(\mathrm{KBr}, \mathrm{cm}^{-1}\right): \widetilde{v}=3326(\mathrm{~s}), 2962(\mathrm{w}), 2947$ (w), $1687(\mathrm{~s}), 1624(\mathrm{w}), 1605(\mathrm{w}), 1578(\mathrm{~m}), 1488(\mathrm{~m}), 1468(\mathrm{~s}), 1441(\mathrm{~s}), 1408(\mathrm{~m}), 1382$ (m), 1306 (m), 1267 (s), 1239 (m), 1224 (m), 1197 (m), 1169 (s), 1133 (w), 1111 (w), 1077 (m), 1048 (m), 955 (w), $867(\mathrm{~m}), 816$ (m), 789 (m), 731 (w), 664 (m), 623 (w). MS (EI, 70 $\mathrm{eV}): m / z(\%)=314\left(\mathrm{M}^{+}\left[{ }^{81} \mathrm{Br}\right], 89\right), 312\left(\mathrm{M}^{+}\left[{ }^{79} \mathrm{Br}\right], 92\right), 283$ (8), 281 (7), 233 (6), 219 (6), 205 (100), 175 (18), 147 (8), 77 (5). The exact molecular mass $\mathrm{m} / \mathrm{z}=311.9997 \pm 2 \mathrm{ppm}\left[\mathrm{M}^{+}\right]$for $\mathrm{C}_{13} \mathrm{H}_{13} \mathrm{O}_{4} \mathrm{Br}$ was confirmed HRMS (EI, $70 \mathrm{eV}$ ). Anal. calcd. for $\mathrm{C}_{13} \mathrm{H}_{13} \mathrm{BrO}_{4}$ (313.147): $\mathrm{C}$, 49.86; H, 4.18; found: C, 50.14; H, 4.88.

Ethyl 2-(3'-Bromopropyl)benzofuran-3-carboxylate (8c): Starting with 7c $(0.080 \mathrm{~g}, 0.30$ mmol $)$ and $\mathrm{BBr}_{3}(0.306 \mathrm{~g}, 1.2 \mathrm{mmol})$ in $\mathrm{CH}_{2} \mathrm{Cl}_{2}(3 \mathrm{~mL}), \mathbf{8 c}$ was isolated after chromatography (silica gel, $n$-hexane/EtOAc $=100: 1 \rightarrow 50: 1)$ as a slightly yellowish oil $(0.078 \mathrm{~g}, 84 \%) .{ }^{1} \mathrm{H}$ NMR ( $\left.\mathrm{CDCl}_{3}, 300 \mathrm{MHz}\right): \delta 1.46\left(\mathrm{t}, J=7.2 \mathrm{~Hz}, 3 \mathrm{H}, \mathrm{CH}_{3}\right), 2.36$ (quint, $J=6.6 \mathrm{~Hz}, 2 \mathrm{H}, \mathrm{CH}_{2}$ ), $3.36\left(\mathrm{t}, J=7.2 \mathrm{~Hz}, 2 \mathrm{H}, \mathrm{CH}_{2}\right), 3.48\left(\mathrm{t}, J=6.6 \mathrm{~Hz}, 2 \mathrm{H}, \mathrm{CH}_{2}-\mathrm{Br}\right), 4.42(\mathrm{q}, J=7.2 \mathrm{~Hz}, 2 \mathrm{H}$, $\left.\mathrm{OCH}_{2}\right), 7.29-7.32(\mathrm{~m}, 2 \mathrm{H}, 2 \times \mathrm{CH}), 7.44-7.47(\mathrm{~m}, 1 \mathrm{H}, \mathrm{CH}), 7.97-8.00(\mathrm{~m}, 1 \mathrm{H}, \mathrm{CH}) .{ }^{13} \mathrm{C}$ $\operatorname{NMR}\left(\mathrm{CDCl}_{3}, 150 \mathrm{MHz}\right): \delta 14.7,27.2,31.1,32.7,60.7,109.6,111.2,122.2,124.1,124.8$, 126.3, 153.9, 164.4, 165.3. IR (neat, $\left.\mathrm{cm}^{-1}\right): \widetilde{v}=2979(\mathrm{w}), 2931(\mathrm{w}), 1711(\mathrm{~s}), 1593(\mathrm{~m}), 1479$ (w), $1451(\mathrm{~m}), 1404(\mathrm{w}), 1380(\mathrm{~m}), 1347(\mathrm{w}), 1283(\mathrm{w}), 1234(\mathrm{~s}), 1176(\mathrm{~m}), 1128(\mathrm{w}), 1104$ (w), 1070 (s), 1012 (w), $789(\mathrm{w}), 751$ (m). MS (EI, $70 \mathrm{eV}): \mathrm{m} / \mathrm{z}(\%)=312\left(\mathrm{M}^{+}\left[{ }^{81} \mathrm{Br}\right], 88\right), 310$ $\left(\mathrm{M}^{+}\left[{ }^{79} \mathrm{Br}\right], 95\right), 283$ (17), 281 (17), 267 (13), 265 (14), 231 (7), 217 (3), 203 (27), 201 (27), 189 (4), 175 (100), 157 (36), 131 (21), 129 (15), 91 (15), 77 (13). HRMS (FT-ICR): Calcd. for $\mathrm{C}_{14} \mathrm{H}_{15} \mathrm{BrO}_{3}\left[\mathrm{M}^{+}\right]$: $313.02623\left({ }^{81} \mathrm{Br}\right), 311.02828\left({ }^{79} \mathrm{Br}\right)$; found: $313.02576\left({ }^{81} \mathrm{Br}\right)$, $311.02779\left({ }^{79} \mathrm{Br}\right)$. 
Ethyl 2-(3'-Bromopropyl)-6-hydroxybenzofuran-3-carboxylate (8d): Starting with 7d $(0.080 \mathrm{~g}, 0.27 \mathrm{mmol})$ and $\mathrm{BBr}_{3}(0.549 \mathrm{~g}, 2.2 \mathrm{mmol})$ in $\mathrm{CH}_{2} \mathrm{Cl}_{2}(3 \mathrm{~mL}), \mathbf{8 d}$ was isolated after chromatography (silica gel, $n$-hexane/EtOAc $=100: 1 \rightarrow 5: 1)$ as a white solid $(0.085 \mathrm{~g}, 97 \%)$, mp. $=132{ }^{\circ} \mathrm{C} .{ }^{1} \mathrm{H} \mathrm{NMR}\left(\mathrm{CDCl}_{3}, 300 \mathrm{MHz}\right): \delta 1.44\left(\mathrm{t}, J=7.2 \mathrm{~Hz}, 3 \mathrm{H}, \mathrm{CH}_{3}\right), 2.33$ (quint, $J=$ $\left.7.05 \mathrm{~Hz}, 2 \mathrm{H}, \mathrm{CH}_{2}\right), 3.31$ (t, $J=7.5 \mathrm{~Hz}, 2 \mathrm{H}, \mathrm{CH}_{2}$ ), 3.47 (t, $J=6.6 \mathrm{~Hz}, 2 \mathrm{H}, \mathrm{CH}_{2}-\mathrm{Br}$ ), 4.41 (q, $\left.J=7.2 \mathrm{~Hz}, 2 \mathrm{H}, \mathrm{OCH}_{2}\right), 4.97($ broad s, $1 \mathrm{H}, \mathrm{OH}), 6.84(\mathrm{dd}, J=8.7,2.1 \mathrm{~Hz}, 1 \mathrm{H}, \mathrm{CH}), 6.94(\mathrm{~d}$, $J=2.1 \mathrm{~Hz}, 1 \mathrm{H}, \mathrm{CH}), 7.80(\mathrm{~d}, J=8.7 \mathrm{~Hz}, 1 \mathrm{H}, \mathrm{CH}) .{ }^{13} \mathrm{C} \mathrm{NMR}\left(\mathrm{CDCl}_{3}+\mathrm{D}_{6}-\mathrm{DMSO}, 150\right.$ $\mathrm{MHz}): \delta 14.3,26.7,30.8,32.6,60.1,97.8,109.1,113.1,118.0,121.7,154.6,155.5,163.3$, 164.2. IR (KBr, $\left.\mathrm{cm}^{-1}\right): \widetilde{v}=3304$ (s), 2987 (w), 1676 (s), 1628 (m), 1594 (m), 1510 (w), 1495 (w), 1443 (s), 1406 (w), 1367 (m), $1301(\mathrm{~m}), 1284(\mathrm{~m}), 1262(\mathrm{~m}), 1247(\mathrm{~m}), 1196(\mathrm{~m}), 1140$ (m), 1113 (s), 1076 (s), $950(w), 838(w), 817$ (w). MS (EI, $70 \mathrm{eV}): \mathrm{m} / \mathrm{z}(\%)=328\left(\mathrm{M}^{+}\left[{ }^{81} \mathrm{Br}\right]\right.$, 97), $326\left(\mathrm{M}^{+}\left[{ }^{79} \mathrm{Br}\right], 100\right), 299$ (24), 297 (25), 283 (9), 281 (9), 219 (75), 217 (25), 191 (88), 175 (10), 173 (19), 146 (14), 118 (7), 91 (7), 77 (6). The exact molecular mass $\mathrm{m} / \mathrm{z}=$ $326.0154 \pm 2 \mathrm{ppm}\left[\mathrm{M}^{+}\right]$for $\mathrm{C}_{14} \mathrm{H}_{15} \mathrm{O}_{4} \mathrm{Br}$ was confirmed by HRMS (EI, $70 \mathrm{eV}$ ). Anal. calcd. for $\mathrm{C}_{14} \mathrm{H}_{15} \mathrm{BrO}_{4}$ (327.174): C, 51.40; H, 4.62; found: C, 51.30; H, 4.97.

Ethyl 2-(3'-Bromopropyl)-4-hydroxybenzofuran-3-carboxylate (8e): Starting with 7e $(0.070 \mathrm{~g}, 0.24 \mathrm{mmol})$ and $\mathrm{BBr}_{3}(0.480 \mathrm{~g}, 1.92 \mathrm{mmol})$ in $\mathrm{CH}_{2} \mathrm{Cl}_{2}(3 \mathrm{~mL})$, 8e was isolated after chromatography (silica gel, $n$-hexane/EtOAc $=100: 1 \rightarrow 50: 1)$ as a white solid $(0.073 \mathrm{~g}$, 92\%); mp. $=86{ }^{\circ} \mathrm{C} .{ }^{1} \mathrm{H} \mathrm{NMR}\left(\mathrm{CDCl}_{3}, 300 \mathrm{MHz}\right): \delta 1.40\left(\mathrm{t}, J=7.2 \mathrm{~Hz}, 3 \mathrm{H}, \mathrm{CH}_{3}\right), 2.27$ (quint, $\left.J=7.05 \mathrm{~Hz}, 2 \mathrm{H}, \mathrm{CH}_{2}\right), 3.23\left(\mathrm{t}, J=7.5 \mathrm{~Hz}, 2 \mathrm{H}, \mathrm{CH}_{2}\right), 3.41$ (t, $\left.J=6.6 \mathrm{~Hz}, 2 \mathrm{H}, \mathrm{CH}_{2}-\mathrm{Br}\right), 4.41$ $\left(\mathrm{q}, J=7.2 \mathrm{~Hz}, 2 \mathrm{H}, \mathrm{OCH}_{2}\right), 6.70(\mathrm{dd}, J=8.1,0.9 \mathrm{~Hz}, 1 \mathrm{H}, \mathrm{CH}), 6.88(\mathrm{dd}, J=8.1 \mathrm{~Hz}, 0.9 \mathrm{~Hz}, 1$ $\mathrm{H}, \mathrm{CH}), 7.12(\mathrm{t}, J=8.1 \mathrm{~Hz}, 1 \mathrm{H}, \mathrm{CH}), 10.40(\mathrm{~s}, 1 \mathrm{H}, \mathrm{OH}) \cdot{ }^{13} \mathrm{C} \mathrm{NMR}\left(\mathrm{CDCl}_{3}, 150 \mathrm{MHz}\right): \delta$ $14.5,27.6,31.0,32.7,62.5,102.4,109.5,110.3,113.7,126.9,151.5,155.3,163.8,167.4$. IR $\left(\mathrm{KBr}, \mathrm{cm}^{-1}\right): \widetilde{v}=3417(\mathrm{w}), 3146(\mathrm{w}), 3126(\mathrm{w}), 3068(\mathrm{w}), 3043(\mathrm{w}), 2974(\mathrm{w}), 2933(\mathrm{w})$, 1667 (s), 1630 (m), 1595 (m), 1481 (m), 1420 (m), 1384 (m), 1353 (w), $1328(\mathrm{w}), 1284(\mathrm{~m})$, 
$1240(\mathrm{~m}), 1201(\mathrm{~m}), 1155(\mathrm{w}), 1084(\mathrm{~m}), 1045(\mathrm{~m}), 1022(\mathrm{w}), 769(\mathrm{~m}), 750(\mathrm{~m}), 728(\mathrm{w}) . \mathrm{MS}$ $(\mathrm{EI}, 70 \mathrm{eV}): \mathrm{m} / \mathrm{z}(\%)=328\left(\mathrm{M}^{+}\left[{ }^{81} \mathrm{Br}\right], 61\right), 326\left(\mathrm{M}^{+}\left[{ }^{79} \mathrm{Br}\right], 62\right), 282$ (100), 280 (100), $226(4)$, 224 (4), 219 (2), 200 (7), 187 (4), 173 (38), 146 (6), 117 (9), 89 (16), 77 (6). The exact molecular mass $m / z=326.0154 \pm 2 \mathrm{ppm}\left[\mathrm{M}^{+}\right]$for $\mathrm{C}_{14} \mathrm{H}_{15} \mathrm{O}_{4} \mathrm{Br}$ was confirmed by HRMS (EI, $70 \mathrm{eV}$ ). Anal. calcd. for $\mathrm{C}_{14} \mathrm{H}_{15} \mathrm{BrO}_{4}$ (327.174): C, 51.40; H, 4.62; found: $\mathrm{C}, 52.15 ; \mathrm{H}, 4.73$.

3-Benzoyl-2-(3'-bromopropyl)benzofuran (8f): Starting with $7 f$ ( $(0.050 \mathrm{~g}, 0.17 \mathrm{mmol})$ and $\mathrm{BBr}_{3}(0.171 \mathrm{~g}, 0.68 \mathrm{mmol})$ in $\mathrm{CH}_{2} \mathrm{Cl}_{2}(3 \mathrm{~mL}), \mathbf{8 f}$ was isolated after chromatography (silica gel, $n$-hexane/EtOAc $=100: 1 \rightarrow 1: 1)$ as a slightly yellowish oil $(0.036 \mathrm{~g}, 71 \%) .{ }^{1} \mathrm{H}$ NMR $\left(\mathrm{CDCl}_{3}, 300 \mathrm{MHz}\right): \delta 2.35$ (quint, $\left.J=7.1 \mathrm{~Hz}, 2 \mathrm{H}, \mathrm{CH}_{2}\right), 3.10\left(\mathrm{t}, J=7.4 \mathrm{~Hz}, 2 \mathrm{H}, \mathrm{CH}_{2}\right), 3.44$ (t, $\left.J=6.8 \mathrm{~Hz}, 2 \mathrm{H}, \mathrm{CH}_{2}-\mathrm{Br}\right), 7.16-7.40(\mathrm{~m}, 3 \mathrm{H}, 3 \times \mathrm{CH}), 7.47-7.64(\mathrm{~m}, 4 \mathrm{H}, 4 \times \mathrm{CH}), 7.81-$ $7.84(\mathrm{~m}, 2 \mathrm{H}, 2 \times \mathrm{CH}) .{ }^{13} \mathrm{C} \mathrm{NMR}\left(\mathrm{CDCl}_{3}, 75 \mathrm{MHz}\right): \delta 27.0,31.0,32.4,111.1,117.4,121.5$, 123.7, 124.6, 126.6, $128.6(2 \mathrm{C}), 129.2$ (2C), 132.9, 139.1, 153.7, 163.5, 191.9. IR (neat, $\mathrm{cm}^{-}$ $\left.{ }^{1}\right): \widetilde{v}=2968(\mathrm{~m}), 2925(\mathrm{~s}), 2857(\mathrm{~m}), 1730(\mathrm{~m}), 1652(\mathrm{~s}), 1573(\mathrm{~m}), 1451(\mathrm{~s}), 1377(\mathrm{~m}), 1281$ (m), 1242 (m), $1178(\mathrm{~m}), 1117(\mathrm{~s}), 1075(\mathrm{w}), 1017(\mathrm{w}), 937(\mathrm{w}), 899(\mathrm{w}), 753(\mathrm{~m}), 700(\mathrm{w})$. MS (EI, $70 \mathrm{eV}): m / z(\%)=344\left(\mathrm{M}^{+}\left[{ }^{81} \mathrm{Br}\right], 29\right), 342\left(\mathrm{M}^{+}\left[{ }^{79} \mathrm{Br}\right], 30\right), 263$ (17), 249 (40), 235 (90), 221 (15), 205 (10), 178 (13), 160 (10), 148 (100), 131 (12), 105 (44), 77 (32). HRMS (ESI): Calcd. for $\mathrm{C}_{18} \mathrm{H}_{15} \mathrm{BrO}_{2}\left[\mathrm{M}^{+}\right]$: $344.02349\left({ }^{81} \mathrm{Br}\right), 342.02554\left({ }^{79} \mathrm{Br}\right)$; found: 344.02303 $\left({ }^{81} \mathrm{Br}\right), 342.02576\left({ }^{79} \mathrm{Br}\right)$.

Methyl 2-(3'-Bromo-4'-chlorobutyl)benzofuran-3-carboxylate (8g): Starting with $7 \mathrm{~g}$ $(0.050 \mathrm{~g}, 0.17 \mathrm{mmol})$ and $\mathrm{BBr}_{3}(0.171 \mathrm{~g}, 0.67 \mathrm{mmol})$ in $\mathrm{CH}_{2} \mathrm{Cl}_{2}(3 \mathrm{~mL}), \mathbf{8 g}$ was isolated after chromatography (silica gel, $n$-hexane/EtOAc $=100: 1 \rightarrow 10: 1$ ) as a slightly yellowish oil (0.047 g, 80\%). ${ }^{1} \mathrm{H}$ NMR $\left(\mathrm{CDCl}_{3}, 300 \mathrm{MHz}\right): \delta 2.21-2.34\left(\mathrm{~m}, 1 \mathrm{H}, \mathrm{CH}_{2}\right), 2.57-2.69(\mathrm{~m}, 1$ $\left.\mathrm{H}, \mathrm{CH}_{2}\right), 3.31-3.42\left(\mathrm{~m}, 1 \mathrm{H}, \mathrm{CH}_{2}\right), 3.44-3.54\left(\mathrm{~m}, 1 \mathrm{H}, \mathrm{CH}_{2}\right), 3.80(\mathrm{dd}, J=11.4,8.4 \mathrm{~Hz}, 1$ $\left.\mathrm{H}, \mathrm{CH}_{2}-\mathrm{Cl}\right), 3.97\left(\mathrm{dd}, J=11.4,4.8 \mathrm{~Hz}, 1 \mathrm{H}, \mathrm{CH}_{2}-\mathrm{Cl}\right), 3.97\left(\mathrm{~s}, 3 \mathrm{H}, \mathrm{OCH}_{3}\right), 4.14-4.22(\mathrm{~m}, 1$ 
$\mathrm{H}, \mathrm{CH}-\mathrm{Br}), 7.30-7.33$ (m, $2 \mathrm{H}, 2 \times \mathrm{CH}$ of $\mathrm{Ar}), 7.44-7.48$ (m, 1 H, CH of Ar), $7.96-7.99$ (m, $1 \mathrm{H}, \mathrm{CH}$ of $\mathrm{Ar}) .{ }^{13} \mathrm{C} \mathrm{NMR}\left(\mathrm{CDCl}_{3}, 75 \mathrm{MHz}\right): \delta 26.0,33.5,48.0,51.6,51.9,109.4,111.0$, 122.0, 124.0, 124.7, 125.9, 153.9, 164.6, 164.8. IR (neat, $\left.\mathrm{cm}^{-1}\right): \widetilde{v}=2952(\mathrm{w}), 2926(\mathrm{w}), 1714$ (s), $1594(\mathrm{~m}), 1446(\mathrm{~m}), 1383(\mathrm{w}), 1285$ (w), 1237 (s), $1176(\mathrm{~m}), 1106(\mathrm{w}), 1070(\mathrm{~s}), 794(\mathrm{w})$, 751 (m). MS (EI, $70 \mathrm{eV}): \mathrm{m} / \mathrm{z}(\%)=348\left(\mathrm{M}^{+}\left[{ }^{81} \mathrm{Br}^{37} \mathrm{Cl}\right], 6\right), 346\left(\mathrm{M}^{+}\left[{ }^{81} \mathrm{Br}^{35} \mathrm{Cl}\right], 34\right), 344\left(\mathrm{M}^{+}\right.$ $\left.\left[{ }^{79} \mathrm{Br}^{35} \mathrm{Cl}\right], 24\right), 315$ (2), 313 (1), 229 (18), 203 (24), 189 (100), 171 (28), 169 (13), 159 (22), 131 (12), 114 (8), 102 (13), 77 (4). HRMS (ESI): Calcd. for $\mathrm{C}_{14} \mathrm{H}_{14} \mathrm{BrClO}_{3}$ [M $\left.{ }^{+}\right]: 345.97944$ $\left({ }^{81} \mathrm{Br}\right), 343.98148\left({ }^{79} \mathrm{Br}\right)$; found: $345.96002\left({ }^{81} \mathrm{Br}\right), 343.98353\left({ }^{79} \mathrm{Br}\right)$.

\section{Methyl 2-[3'-Bromo-1'-(2'-hydroxyphenyl)propyl]benzofuran-3-carboxylate $\quad(8 \mathrm{~h})$ :}

Starting with $7 \mathbf{h}(0.030 \mathrm{~g}, 0.085 \mathrm{mmol})$ and $\mathrm{BBr}_{3}(0.129 \mathrm{~g}, 0.51 \mathrm{mmol})$ in $\mathrm{CH}_{2} \mathrm{Cl}_{2}(9 \mathrm{~mL}), \mathbf{8 h}$ was isolated after chromatography (silica gel, $n$-hexane/EtOAc $=100: 1 \rightarrow 4: 1$ ) as a colorless oil (0.019 g, 58\%). ${ }^{1} \mathrm{H}$ NMR $\left(\mathrm{CDCl}_{3}, 300 \mathrm{MHz}\right): \delta 2.68-2.81\left(\mathrm{~m}, 1 \mathrm{H}, \mathrm{CH}_{2}\right), 2.84-2.95(\mathrm{~m}$, $\left.1 \mathrm{H}, \mathrm{CH}_{2}\right), 3.25-3.41\left(\mathrm{~m}, 2 \mathrm{H}, \mathrm{CH}_{2}-\mathrm{Br}\right), 4.06\left(\mathrm{~s}, 3 \mathrm{H}, \mathrm{OCH}_{3}\right), 5.53(\mathrm{t}, J=7.6 \mathrm{~Hz}, 1 \mathrm{H}, \mathrm{CH})$, $6.91-6.97(\mathrm{~m}, 2 \mathrm{H}, 2 \times \mathrm{CH}), 7.13-7.19(\mathrm{~m}, 1 \mathrm{H}, \mathrm{CH}), 7.29-7.35(\mathrm{~m}, 2 \mathrm{H}, 2 \times \mathrm{CH}), 7.45-$ $7.50(\mathrm{~m}, 2 \mathrm{H}, 2 \times \mathrm{CH}), 7.86-7.89(\mathrm{~m}, 1 \mathrm{H}, \mathrm{CH}), 7.91(\mathrm{~s}, 1 \mathrm{H}, \mathrm{OH}) .{ }^{13} \mathrm{C} \mathrm{NMR}\left(\mathrm{CDCl}_{3}, 75\right.$ $\mathrm{MHz}): \delta 30.6,35.0,35.3,52.5,111.3,117.9,120.9,121.1,122.1,124.3,124.5,124.8,125.2$, 127.7, 128.8, 153.9, 154.5, 165.5, 167.1. IR (neat, $\mathrm{cm}^{-1}$ ): $\widetilde{v}=3354$ (br), $2974(\mathrm{~m}), 2956(\mathrm{~m})$, 2928 (m), 2858 (w), 1738 (w), 1712 (s), 1688 (s), 1588 (m), 1482 (m), 1454 (s), 1368 (m), 1288 (m), 1239 (s), 1174 (s), 1154 (m), 1108 (s), 1069 (s), 1020 (m), 984 (w), 793 (w), 754 (s). MS (EI, $70 \mathrm{eV}): m / z(\%)=390\left(\mathrm{M}^{+}\left[{ }^{81} \mathrm{Br}\right], 18\right), 388\left(\mathrm{M}^{+}\left[{ }^{79} \mathrm{Br}\right], 19\right), 358(16), 356$ (17), 329 (5), 326 (5), 281 (44), 263 (11), 249 (100), 221 (65), 205 (5), 193 (13), 165 (14), 163 (11), 161 (23), 134 (25), 114 (20), 107 (13), 105 (14), 91 (11), 77 (22). The exact molecular mass $m / z=388.0310 \pm 2 \mathrm{ppm}\left[\mathrm{M}^{+}\right]$for $\mathrm{C}_{19} \mathrm{H}_{17} \mathrm{BrO}_{4}$ was confirmed by HRMS (EI, $70 \mathrm{eV}$ ). 
General procedure for the synthesis of 9 by reaction of 2 -alkylidenetetrahydrofurans with borontribromide: To a $\mathrm{CH}_{2} \mathrm{Cl}_{2}$-solution $(10 \mathrm{~mL} / \mathrm{mmol})$ of the 2alkylidenetetrahydrofuran (7) (1 equiv.) was added $\mathrm{BBr}_{3}$ (4 equiv.) at $0{ }^{\circ} \mathrm{C}$. The reaction mixture was allowed to warm to $20{ }^{\circ} \mathrm{C}$ over $12 \mathrm{~h}$ and was stirred for $12 \mathrm{~h}$ at $20{ }^{\circ} \mathrm{C}$. Then, the reaction mixture was poured into an aqueous solution of $\mathrm{KOtBu}(1 \mathrm{M}, 10 \mathrm{~mL} / \mathrm{mmol})$. The mixture was stirred for $1 \mathrm{~h}$ and later extracted with $\mathrm{CH}_{2} \mathrm{Cl}_{2}(3 \times 30 \mathrm{~mL} / \mathrm{mmol})$. The combined organic extracts were dried $\left(\mathrm{Na}_{2} \mathrm{SO}_{4}\right)$, filtered and the filtrate was concentrated in vacuo. The residue was purified by chromatography (silica gel, $n$-hexane/EtOAc) to give 9 .

3-(Dihydro-furan-2(3H)-ylidene)-3H-benzofuran-2-one (9a): Starting with 7a $(0.070 \mathrm{~g}$, $0.282 \mathrm{mmol})$ and $\mathrm{BBr}_{3}(0.283 \mathrm{~g}, 1.13 \mathrm{mmol})$ in $\mathrm{CH}_{2} \mathrm{Cl}_{2}(3 \mathrm{~mL}), \mathbf{E - 9 a}$ and $\mathbf{Z - 9 a}$ were isolated after chromatography ( silica gel, $n$-hexane/EtOAc $=50: 1 \rightarrow 1: 1)$ as yellowish solids $(0.031 \mathrm{~g}$, $54 \% ; 0.020 \mathrm{~g}, 35 \%)$.

E-9a: mp. $=170{ }^{\circ} \mathrm{C} .{ }^{1} \mathrm{H} \mathrm{NMR}\left(\mathrm{CDCl}_{3}, 300 \mathrm{MHz}\right.$ ): $\delta 2.28$ (quint, $J=7.5 \mathrm{~Hz}, 2 \mathrm{H}, \mathrm{CH}_{2}$ ), 3.37 $\left(\mathrm{t}, J=7.8 \mathrm{~Hz}, 2 \mathrm{H}, \mathrm{CH}_{2}\right), 4.62\left(\mathrm{t}, J=7.2 \mathrm{~Hz}, 2 \mathrm{H}, \mathrm{OCH}_{2}\right), 7.06-7.21(\mathrm{~m}, 3 \mathrm{H}, 3 \times \mathrm{CH}$ of $\mathrm{Ar})$, $7.60(\mathrm{dd}, J=7.2,1.2 \mathrm{~Hz}, 1 \mathrm{H}, \mathrm{CH}$ of $\mathrm{Ar}) .{ }^{13} \mathrm{C} \mathrm{NMR}\left(\mathrm{CDCl}_{3}, 75 \mathrm{MHz}\right): \delta 23.5,31.4,75.0$, $95.9,110.0,122.0,123.5,124.1,126.7,151.0,169.8,175.2 . \operatorname{IR}\left(\mathrm{KBr}, \mathrm{cm}^{-1}\right): \widetilde{v}=2911(\mathrm{w})$, 1750 (s), 1652 (s), $1611(\mathrm{w}), 1589$ (w), 1479 (w), 1459 (m), 1420 (w), 1392 (m), 1247 (s), 1220 (m), 1166 (m), 1032 (m), 977 (s), 939 (m), 866 (m), 780 (m), 751 (m). MS (EI, 70 eV): $m / z(\%)=202\left(\mathrm{M}^{+}, 100\right), 159(10)$. The exact molecular mass $m / z=202.0630 \pm 2 \mathrm{ppm}\left[\mathrm{M}^{+}\right]$ for $\mathrm{C}_{12} \mathrm{H}_{10} \mathrm{O}_{3}$ was confirmed by HRMS (EI, $\left.70 \mathrm{eV}\right)$.

Z-9a: mp. $=106{ }^{\circ} \mathrm{C} .{ }^{1} \mathrm{H} \mathrm{NMR}\left(\mathrm{CDCl}_{3}, 300 \mathrm{MHz}\right): \delta 2.34$ (quint, $\left.J=7.5 \mathrm{~Hz}, 2 \mathrm{H}, \mathrm{CH}_{2}\right), 3.19$ $\left(\mathrm{t}, J=7.8 \mathrm{~Hz}, 2 \mathrm{H}, \mathrm{CH}_{2}\right), 4.68\left(\mathrm{t}, J=7.2 \mathrm{~Hz}, 2 \mathrm{H}, \mathrm{OCH}_{2}\right), 7.06-7.25(\mathrm{~m}, 4 \mathrm{H}, 4 \times \mathrm{CH}$ of $\mathrm{Ar})$. ${ }^{13} \mathrm{C} \mathrm{NMR}\left(\mathrm{CDCl}_{3}, 75 \mathrm{MHz}\right): \delta 23.1,32.4,75.3,95.9,110.6,119.6,123.4,124.7,126.5,151.3$, 165.9, 172.9. IR $\left(\mathrm{KBr}, \mathrm{cm}^{-1}\right): \widetilde{v}=2958(\mathrm{w}), 2928(\mathrm{w}), 2855(\mathrm{w}), 1814(\mathrm{w}), 1760(\mathrm{~s}), 1638(\mathrm{~s})$, 
1457 (s), 1422 (w), 1392 (w), 1349 (w), 1308 (w), 1288 (w), 1245 (s), $1221(\mathrm{~m}), 1192$ (m), 1158 (w), 1133 (s), 1084 (s), 1028 (s), 997 (m), 976 (w), 924 (s), 868 (w), 776 (m), 750 (s). MS (EI, $70 \mathrm{eV}): m / z(\%)=202\left(\mathrm{M}^{+}, 87\right), 159(100)$. The exact molecular mass $\mathrm{m} / \mathrm{z}=$ $202.0630 \pm 2 \mathrm{ppm}\left[\mathrm{M}^{+}\right]$for $\mathrm{C}_{12} \mathrm{H}_{10} \mathrm{O}_{3}$ was confirmed by HRMS (EI, $70 \mathrm{eV}$ ).

\section{3-(5-Chloromethyl-dihydro-furan-2(3H)-ylidene)-3H-benzofuran-2-one $\quad(9 \mathrm{~b}): \quad$ Starting} with $7 \mathbf{g}(0.050 \mathrm{~g}, 0.17 \mathrm{mmol})$ and $\mathrm{BBr}_{3}(0.171 \mathrm{~g}, 0.67 \mathrm{mmol})$ in $\mathrm{CH}_{2} \mathrm{Cl}_{2}(3 \mathrm{~mL}), \boldsymbol{E}-\mathbf{9 b}$ and $\mathbf{Z}$ 9b were isolated after chromatography (silica gel, $n$-hexane/EtOAc $=100: 1 \rightarrow 1: 1$ ) as yellowish oils $(0.028 \mathrm{~g}, 65 \% ; 0.013 \mathrm{~g}, 30 \%)$

E-9b: mp. $=61{ }^{\circ} \mathrm{C} .{ }^{1} \mathrm{H}$ NMR $\left(\mathrm{CDCl}_{3}, 300 \mathrm{MHz}\right): \delta 2.12-2.23\left(\mathrm{~m}, 1 \mathrm{H}, \mathrm{CH}_{2}\right), 2.39-2.52(\mathrm{~m}$, $\left.1 \mathrm{H}, \mathrm{CH}_{2}\right), 3.25-3.38\left(\mathrm{~m}, 1 \mathrm{H}, \mathrm{CH}_{2}\right), 3.52-3.60\left(\mathrm{~m}, 1 \mathrm{H}, \mathrm{CH}_{2}\right), 3.80(\mathrm{~d}, J=5.1 \mathrm{~Hz}, 1 \mathrm{H}$, $\left.\mathrm{CH}_{2}-\mathrm{Cl}\right), 3.84\left(\mathrm{~d}, J=5.1 \mathrm{~Hz}, 1 \mathrm{H}, \mathrm{CH}_{2}-\mathrm{Cl}\right), 5.02-5.08(\mathrm{~m}, 1 \mathrm{H}, \mathrm{OCH}), 7.07-7.21(\mathrm{~m}, 3 \mathrm{H}$, $3 \times \mathrm{CH}$ of $\mathrm{Ar}), 7.61(\mathrm{dd}, J=7.5,1.2 \mathrm{~Hz}, 1 \mathrm{H}, \mathrm{CH}$ of $\mathrm{Ar}) .{ }^{13} \mathrm{C} \mathrm{NMR}\left(\mathrm{CDCl}_{3}, 75 \mathrm{MHz}\right): \delta 26.1$, 31.0, 45.3, 85.2, 96.3, 109.8, 122.0, 123.4, 126.8, 126.8, 150.8, 169.4, 173.3. IR $\left(\mathrm{KBr}, \mathrm{cm}^{-1}\right)$ : $\widetilde{v}=2976(\mathrm{~s}), 2932(\mathrm{w}), 2866(\mathrm{~m}), 1758(\mathrm{~s}), 1746(\mathrm{~s}), 1655(\mathrm{~s}), 1612(\mathrm{w}), 1455(\mathrm{~s}), 1378(\mathrm{~m})$, 1360 (m), 1296 (w), 1242 (s), 1206 (s), 1151 (s), 1118 (s), 1076 (m), 1015 (s), 964 (s), 933 (w), $874(\mathrm{w}), 832(\mathrm{w}), 752(\mathrm{~s}) . \mathrm{MS}(\mathrm{EI}, 70 \mathrm{eV}): \mathrm{m} / \mathrm{z}(\%)=252\left(\mathrm{M}^{+}\left[{ }^{37} \mathrm{Cl}\right], 20\right), 250\left(\mathrm{M}^{+}\left[{ }^{35} \mathrm{Cl}\right]\right.$, 73), 215 (20), 187 (8), 162 (2), 160 (100), 121 (49), 104 (25), 85 (76), 76 (26). HRMS (ESI): Calcd. for $\mathrm{C}_{13} \mathrm{H}_{11} \mathrm{ClO}_{3}\left[\mathrm{M}^{+}\right]$: $252.03672\left({ }^{37} \mathrm{Cl}\right), 250.03967\left({ }^{35} \mathrm{Cl}\right)$; found: $252.03800\left({ }^{37} \mathrm{Cl}\right)$, $250.03903\left({ }^{35} \mathrm{Cl}\right)$.

Z-9b: ${ }^{1} \mathrm{H}$ NMR $\left(\mathrm{CDCl}_{3}, 300 \mathrm{MHz}\right): \delta 2.34-2.39\left(\mathrm{~m}, 1 \mathrm{H}, \mathrm{CH}_{2}\right), 2.45-2.58\left(\mathrm{~m}, 1 \mathrm{H}, \mathrm{CH}_{2}\right)$, $3.14-3.39\left(\mathrm{~m}, 2 \mathrm{H}, \mathrm{CH}_{2}\right), 3.86\left(\mathrm{~d}, J=0.9 \mathrm{~Hz}, 1 \mathrm{H}, \mathrm{CH}_{2}-\mathrm{Cl}\right), 3.87\left(\mathrm{~d}, J=1.8 \mathrm{~Hz}, 1 \mathrm{H}, \mathrm{CH}_{2}-\right.$ $\mathrm{Cl}), 5.13-5.19(\mathrm{~m}, 1 \mathrm{H}, \mathrm{OCH}), 7.08-7.23(\mathrm{~m}, 4 \mathrm{H}, 4 \times \mathrm{CH}$ of $\mathrm{Ar}) .{ }^{13} \mathrm{C} \mathrm{NMR}\left(\mathrm{CDCl}_{3}, 75\right.$ MHz): $\delta 25.5,31.0,45.6,85.1,95.9,110.5,119.6,123.3,124.2,126.6,151.2,167.3,171.6$. IR (KBr, cm $\left.{ }^{-1}\right): \widetilde{v}=2927(\mathrm{w}), 1756(\mathrm{~s}), 1644(\mathrm{~s}), 1459(\mathrm{w}), 1243(\mathrm{~m}), 1140(\mathrm{~m}), 1086(\mathrm{~m})$, 1014 (m), 938 (w), 777 (w), 747 (m). MS (EI, $70 \mathrm{eV}): \mathrm{m} / \mathrm{z}(\%)=252\left(\mathrm{M}^{+}\left[{ }^{37} \mathrm{Cl}\right], 2\right), 250\left(\mathrm{M}^{+}\right.$ 
$\left.\left[{ }^{35} \mathrm{Cl}\right], 12\right), 215$ (6), 187 (4), 160 (27), 145 (3), 131 (3), 121 (12), 104 (12), 85 (100), 77 (16). HRMS (ESI): Calcd. for $\mathrm{C}_{13} \mathrm{H}_{11} \mathrm{ClO}_{3}\left[\mathrm{M}^{+}\right]$: $252.03672\left({ }^{37} \mathrm{Cl}\right), 250.03967\left({ }^{35} \mathrm{Cl}\right)$; found: $252.03800\left({ }^{37} \mathrm{Cl}\right), 250.03903\left({ }^{35} \mathrm{Cl}\right)$. 

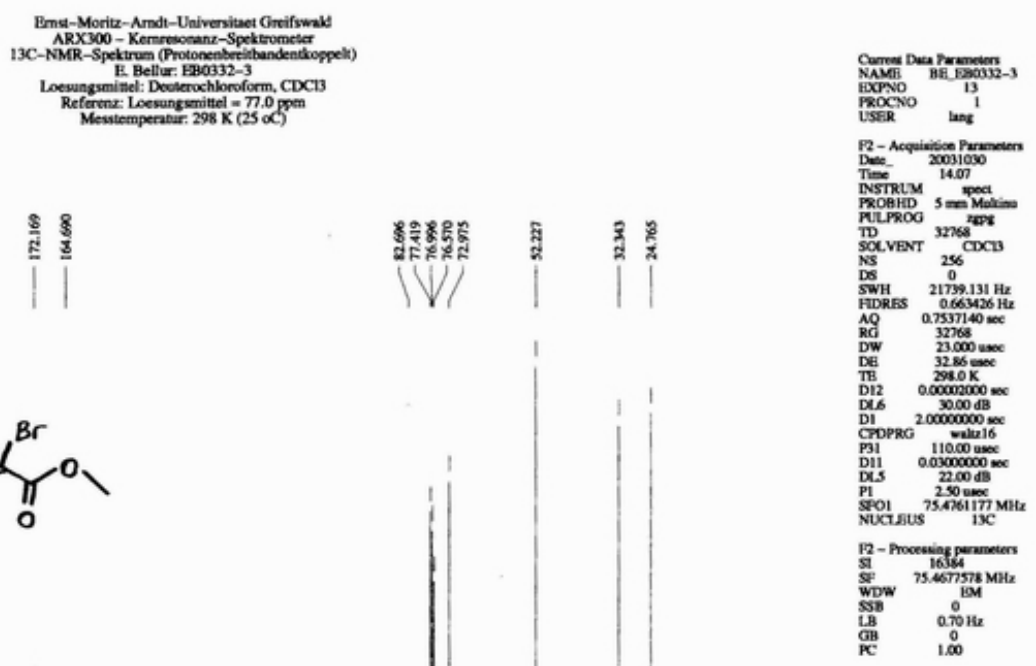

(3a)

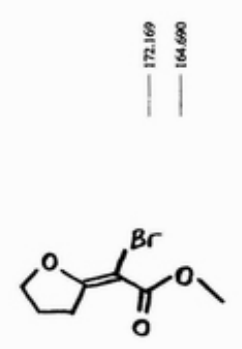

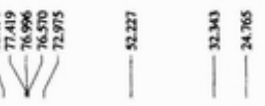

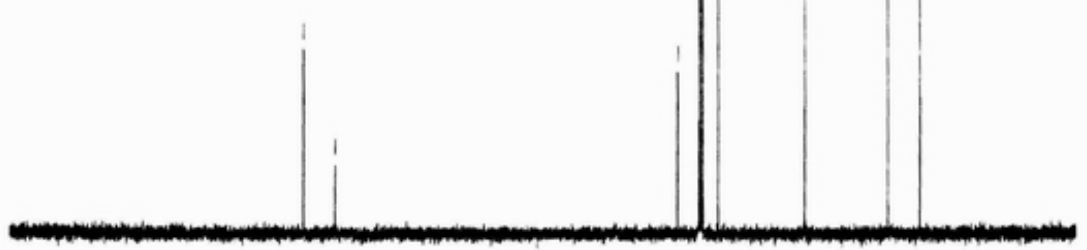

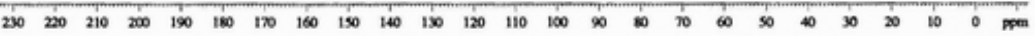
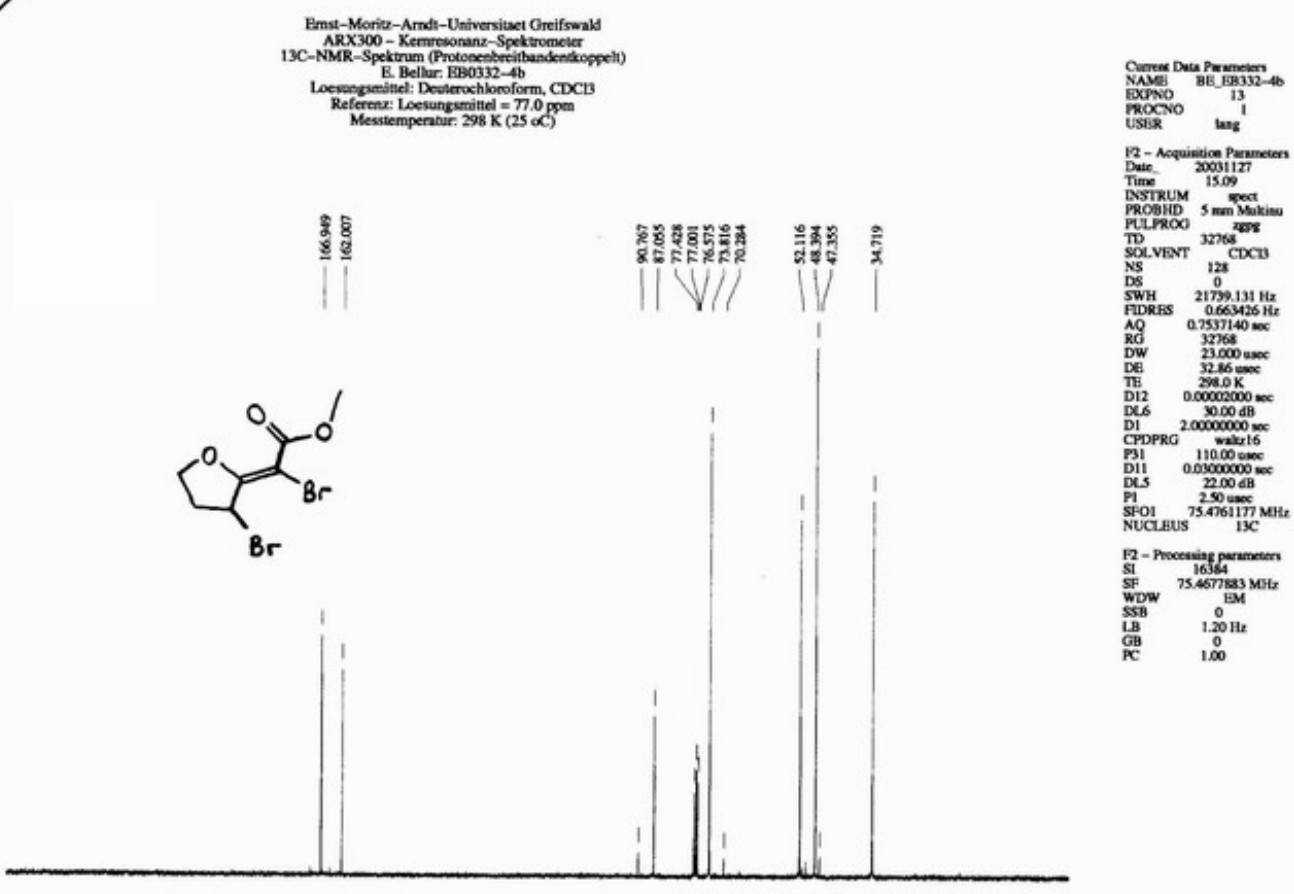

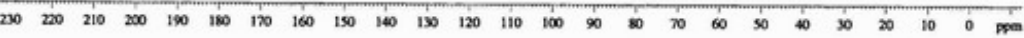



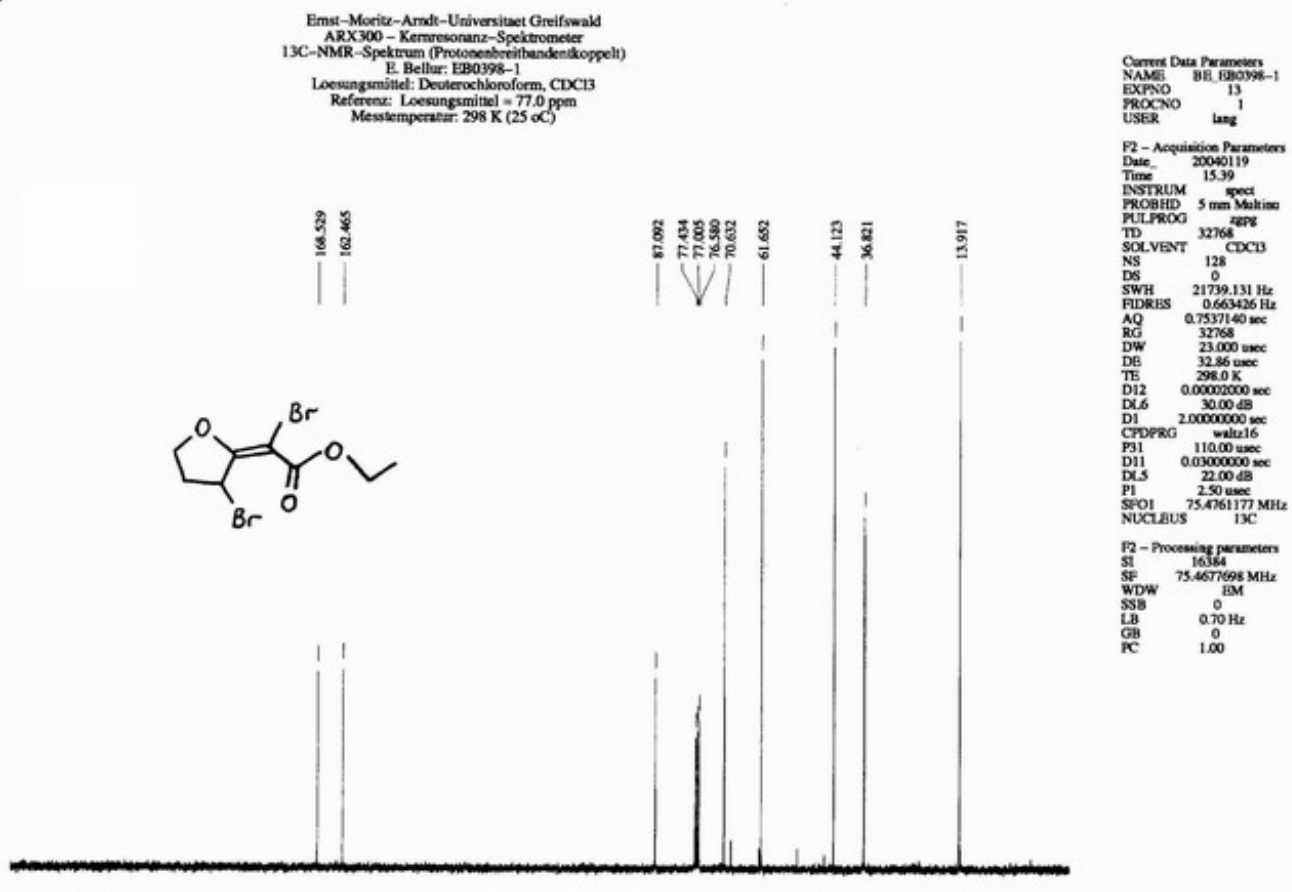

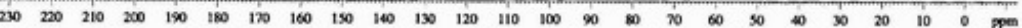

BB 0381-2a

(3b)
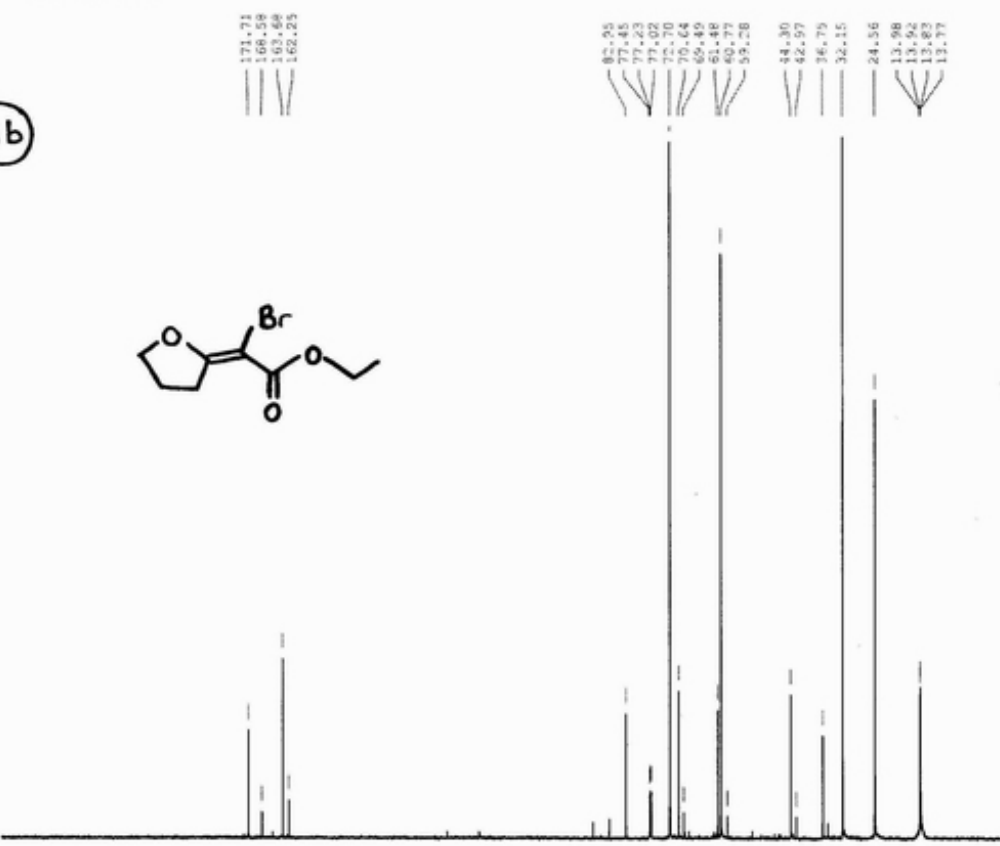
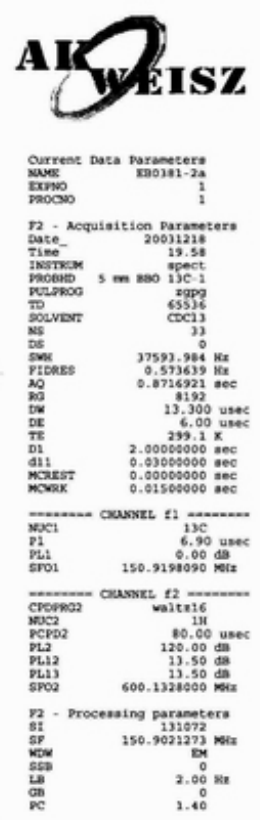

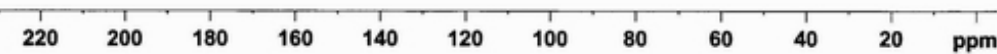


EB 0381-3a
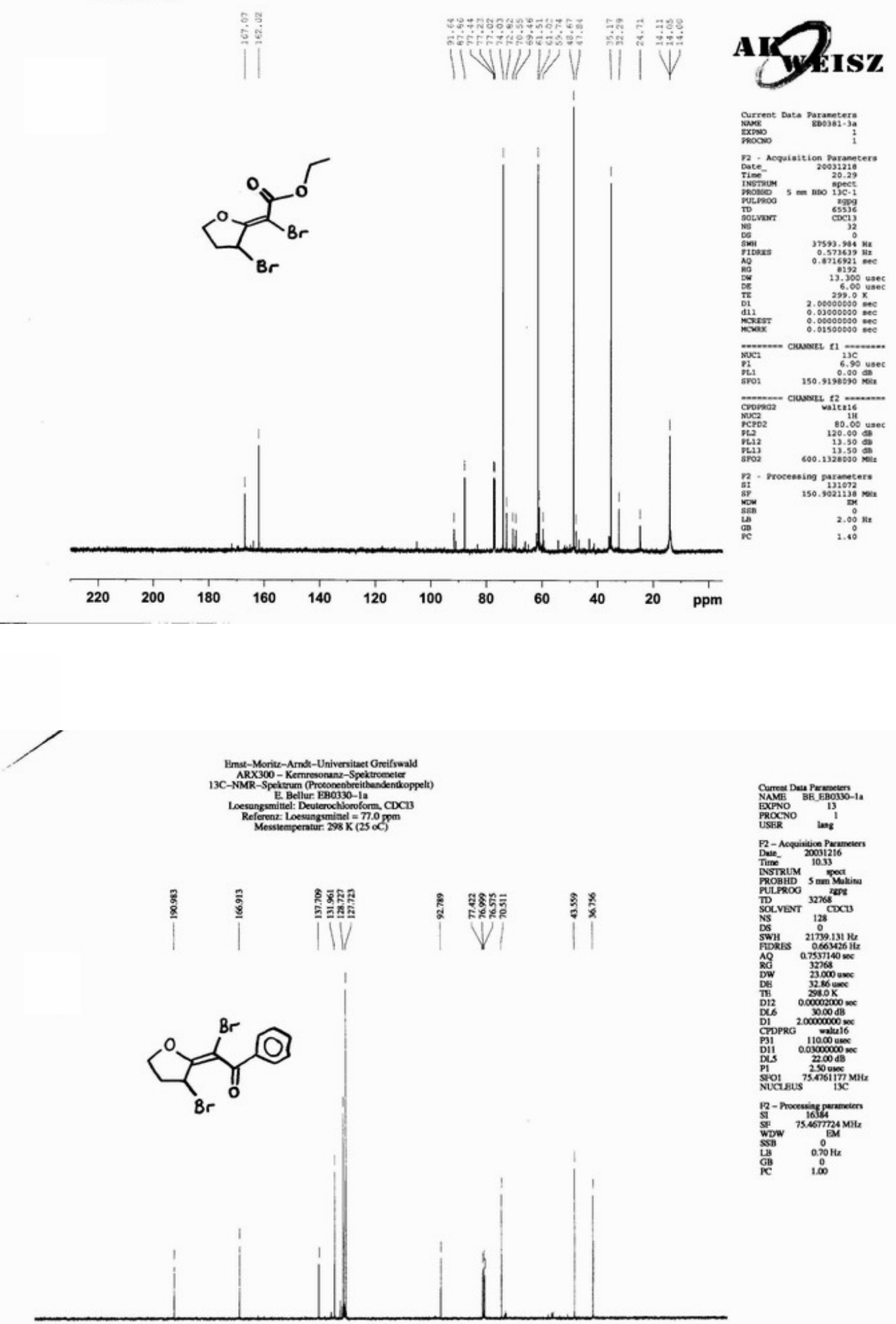

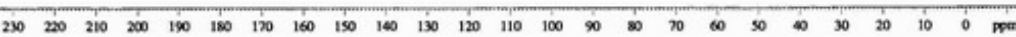


(3c)
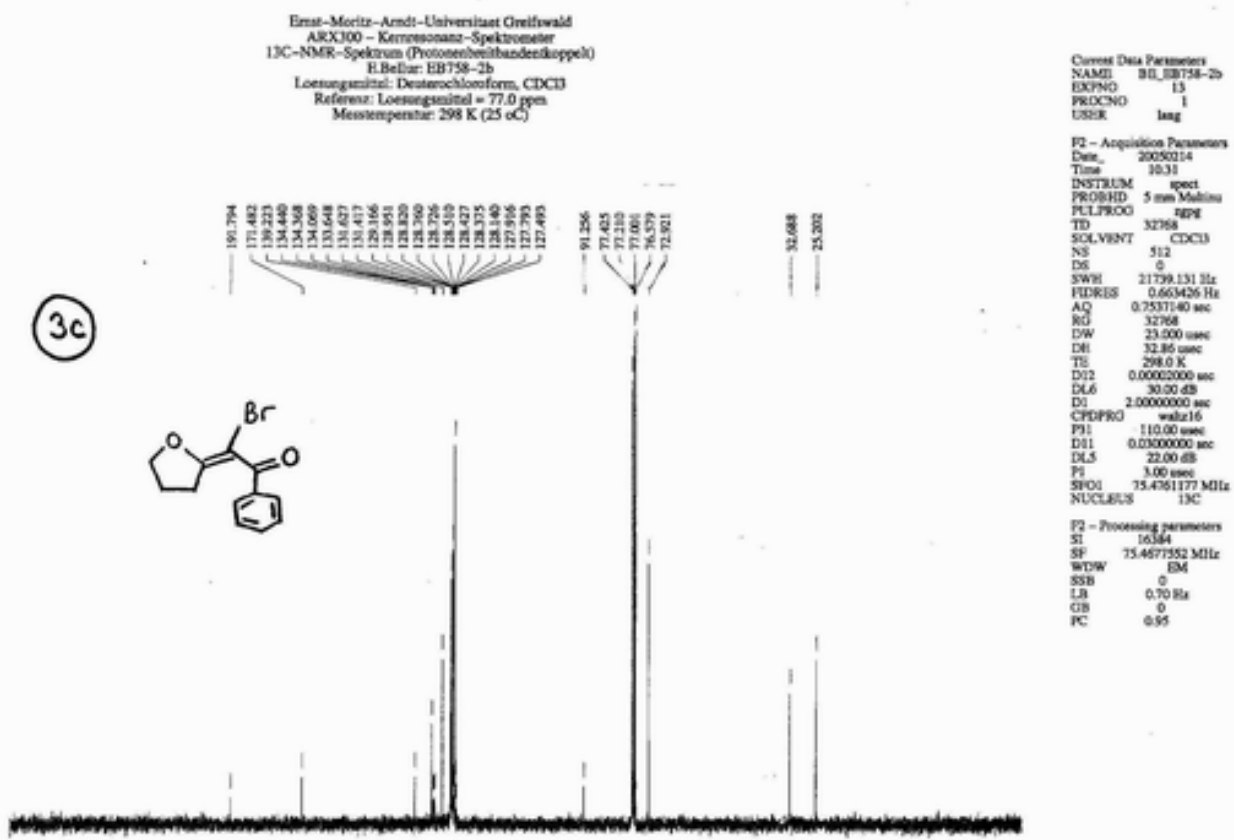

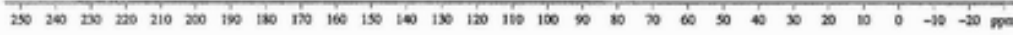
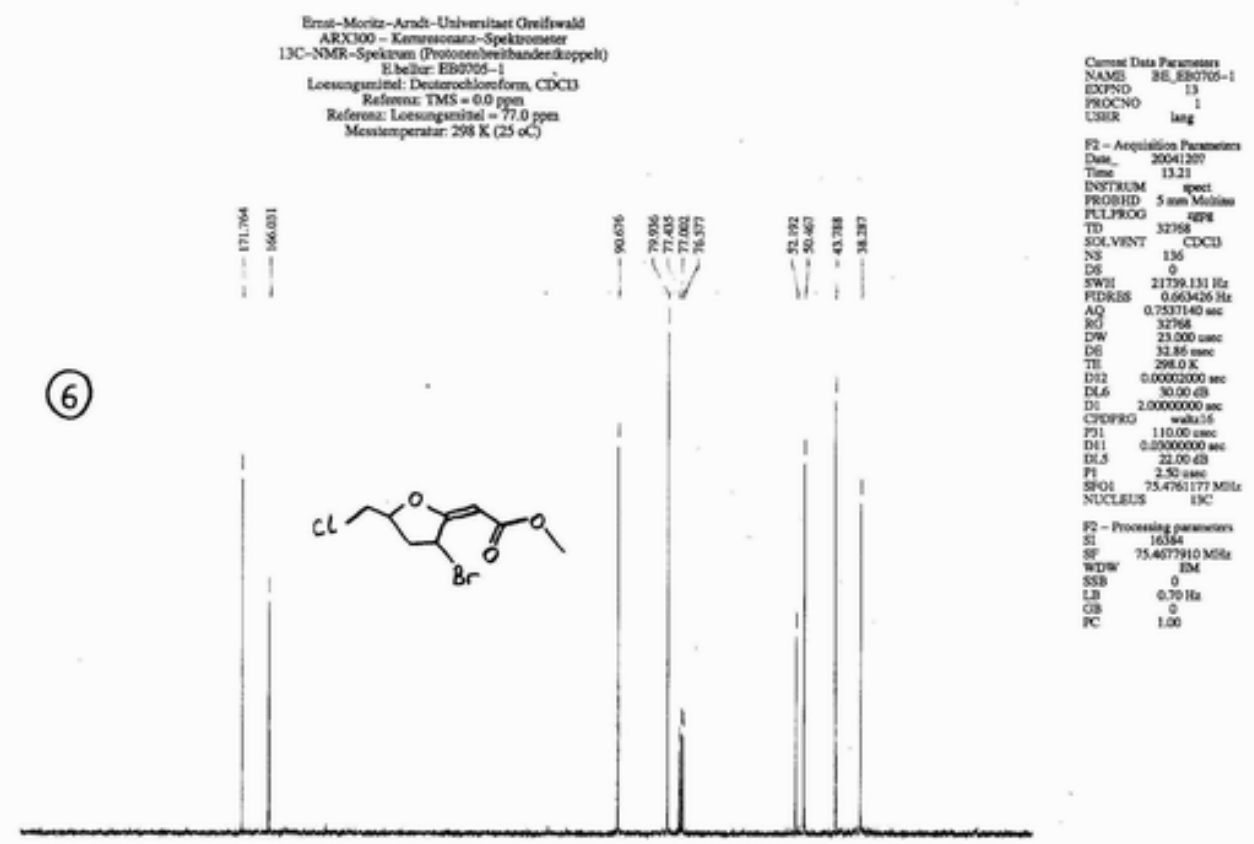

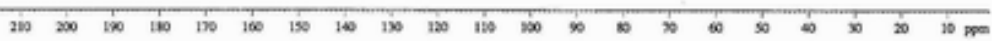




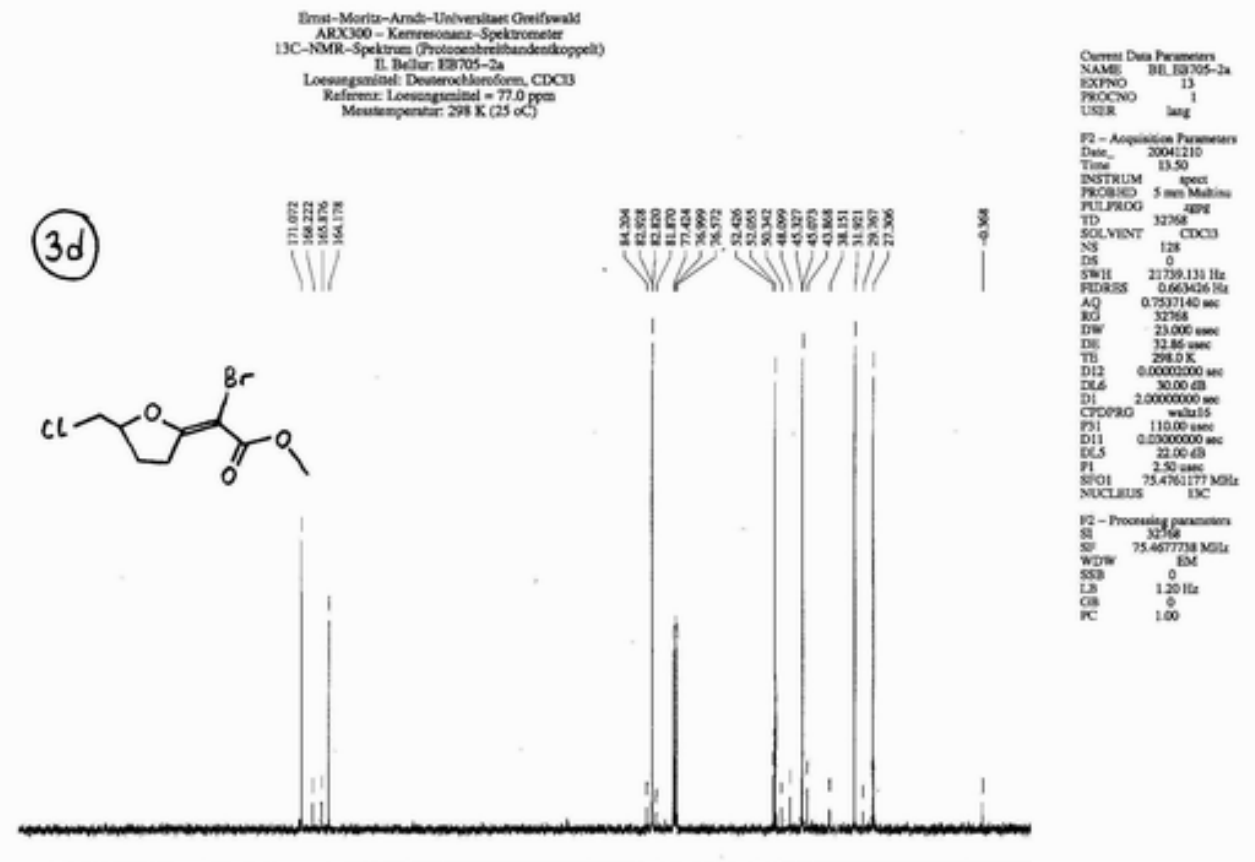

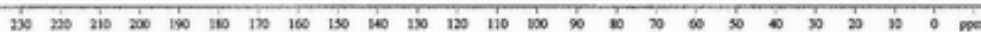

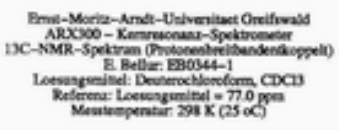

(7a)

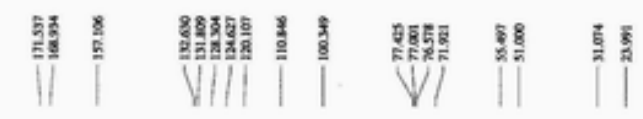<smiles>COC(=O)C1C2CCC1(C)OC21CCCO1</smiles>
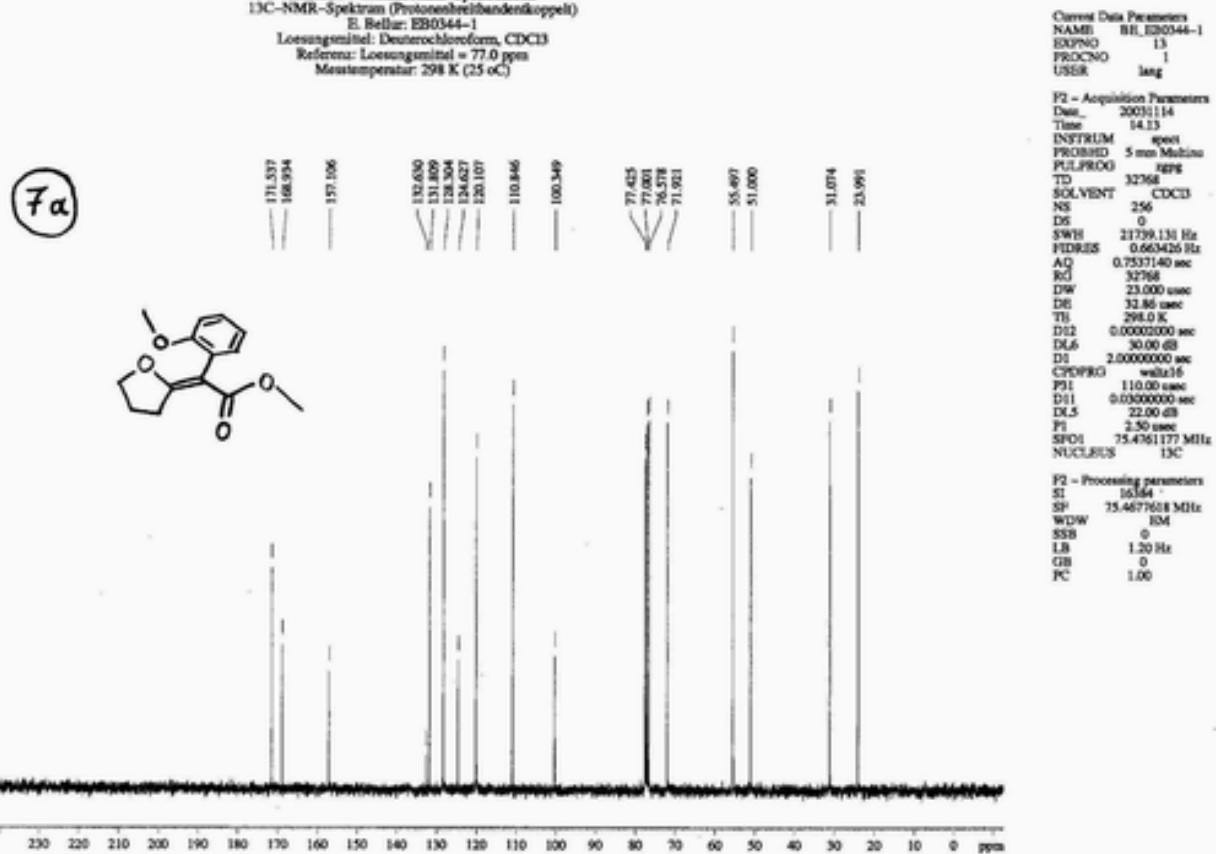


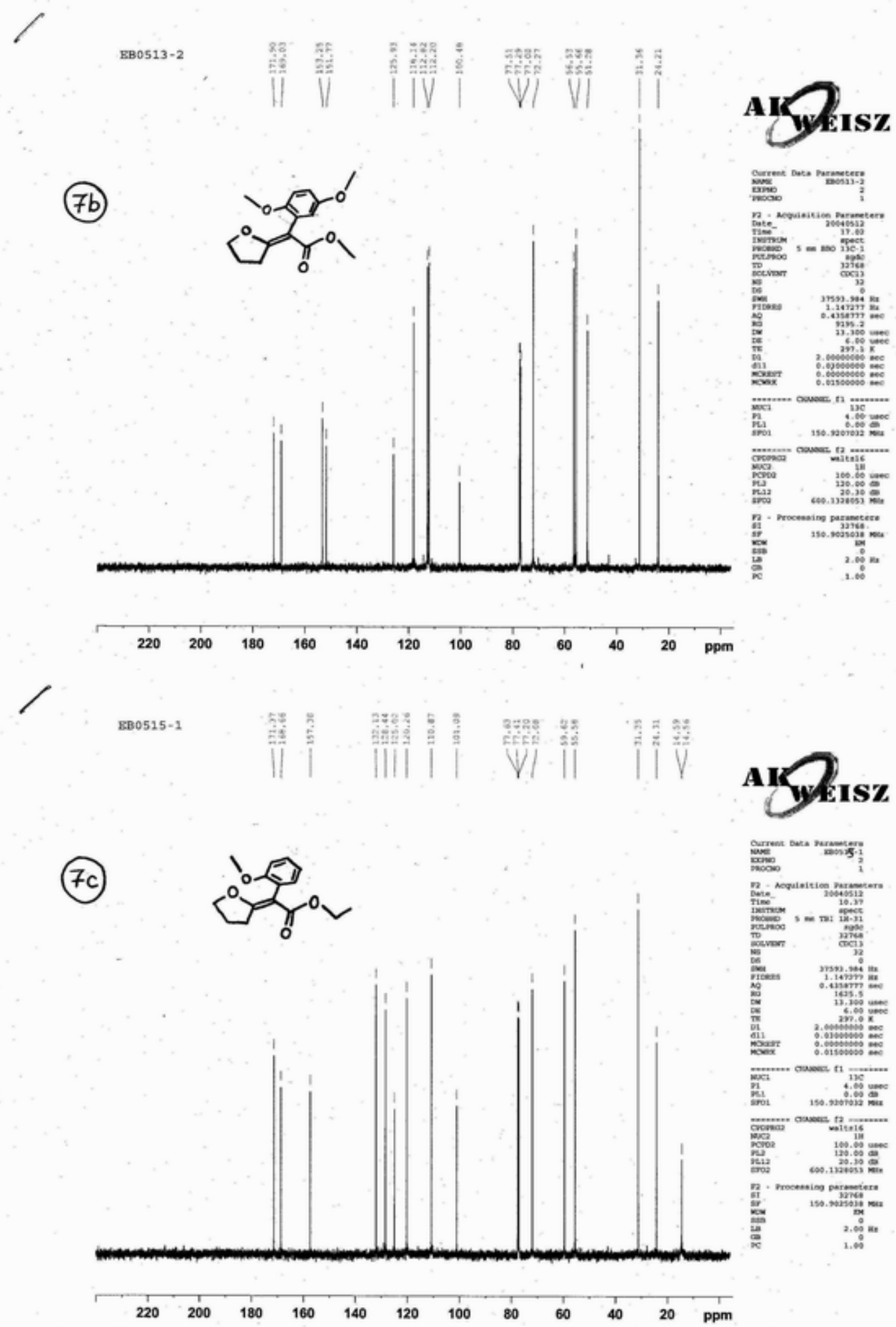



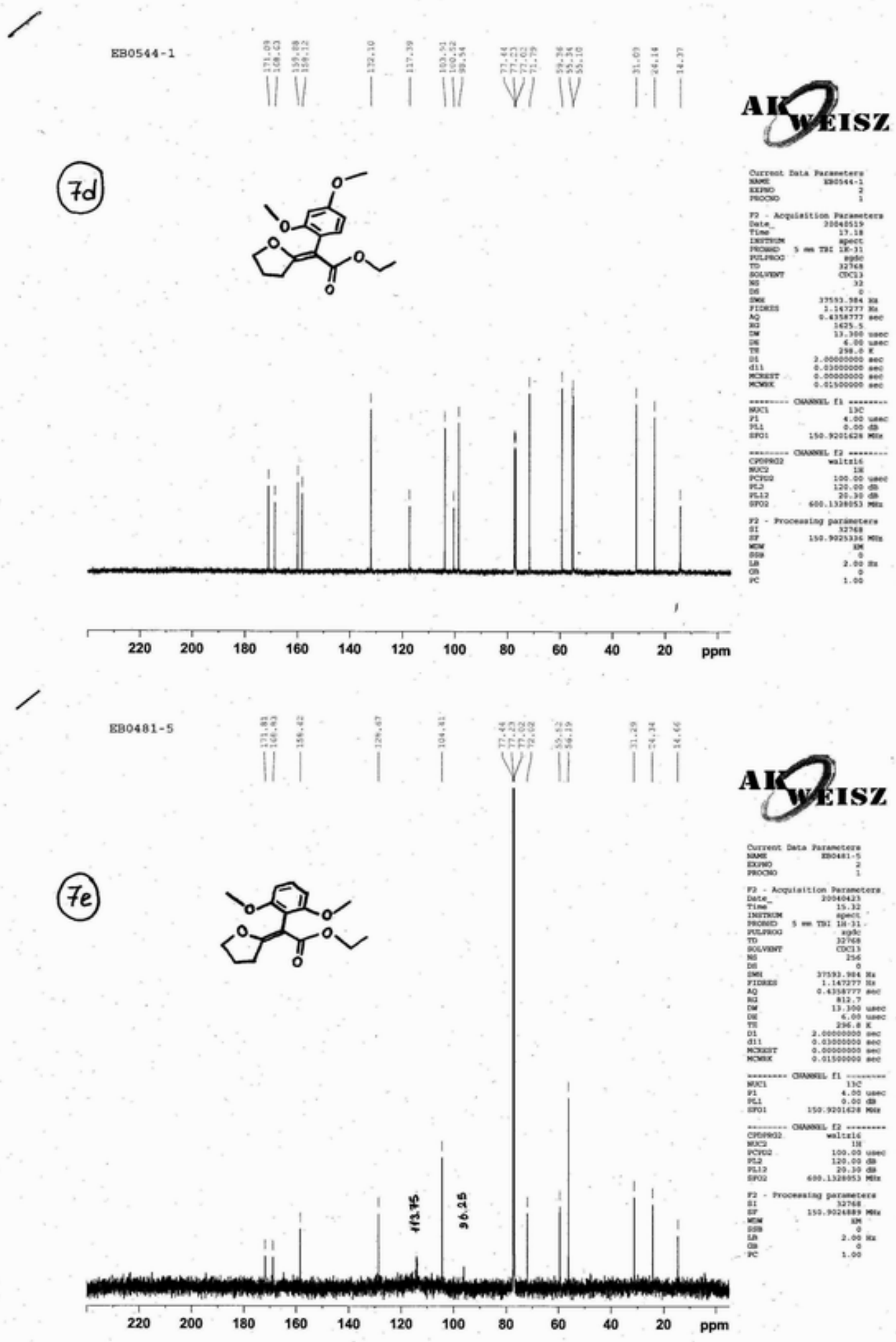
13C EBonz-2 in $\mathrm{COCC}_{13}$
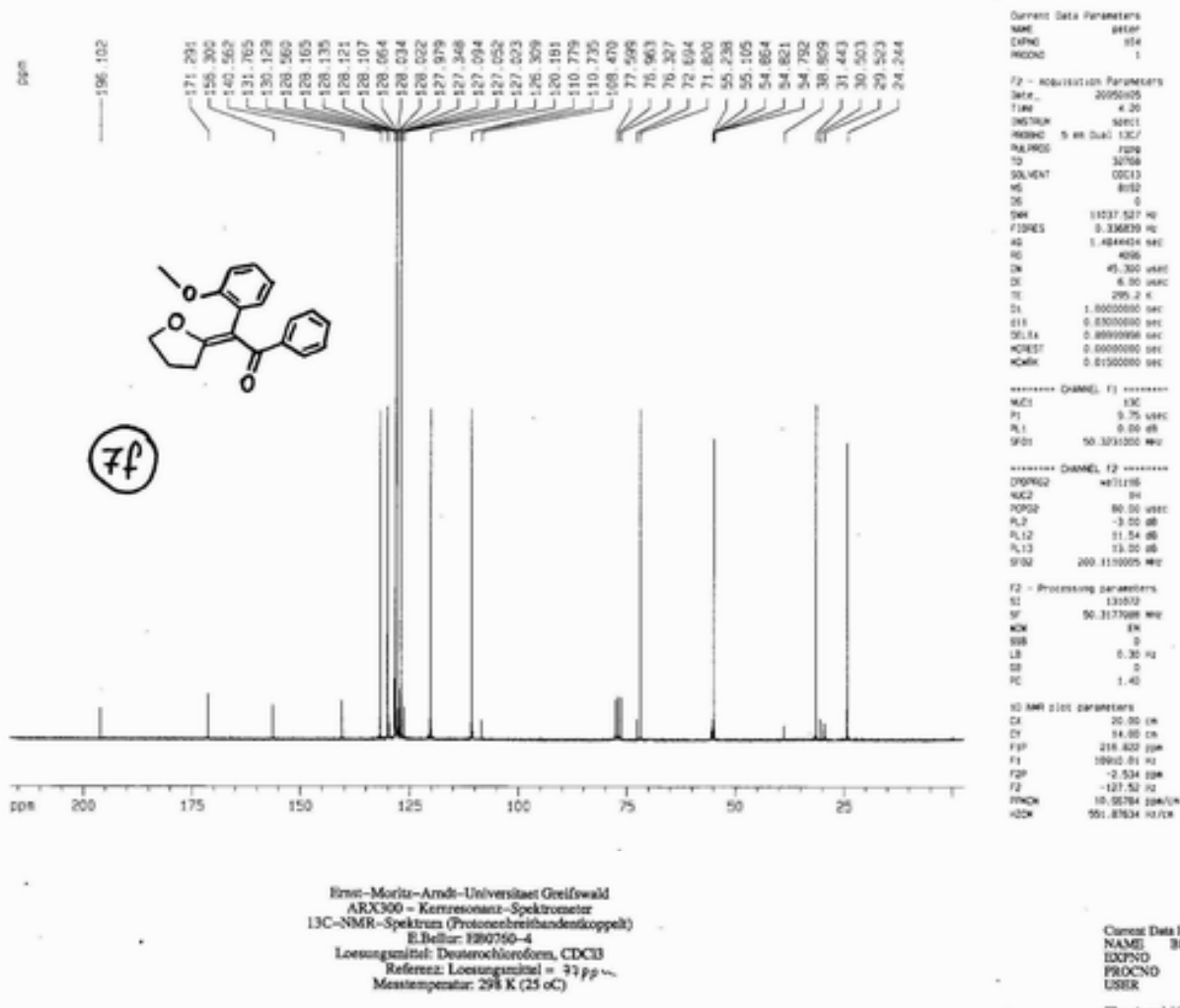

(79)
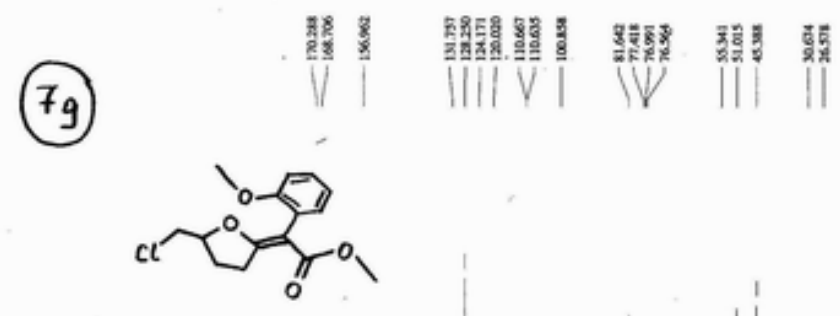

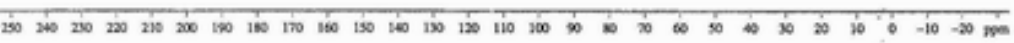

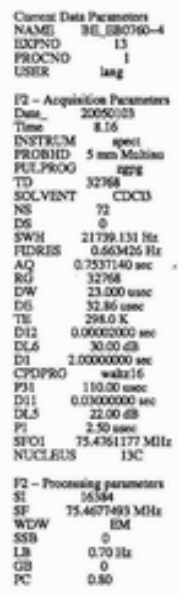


(7h)
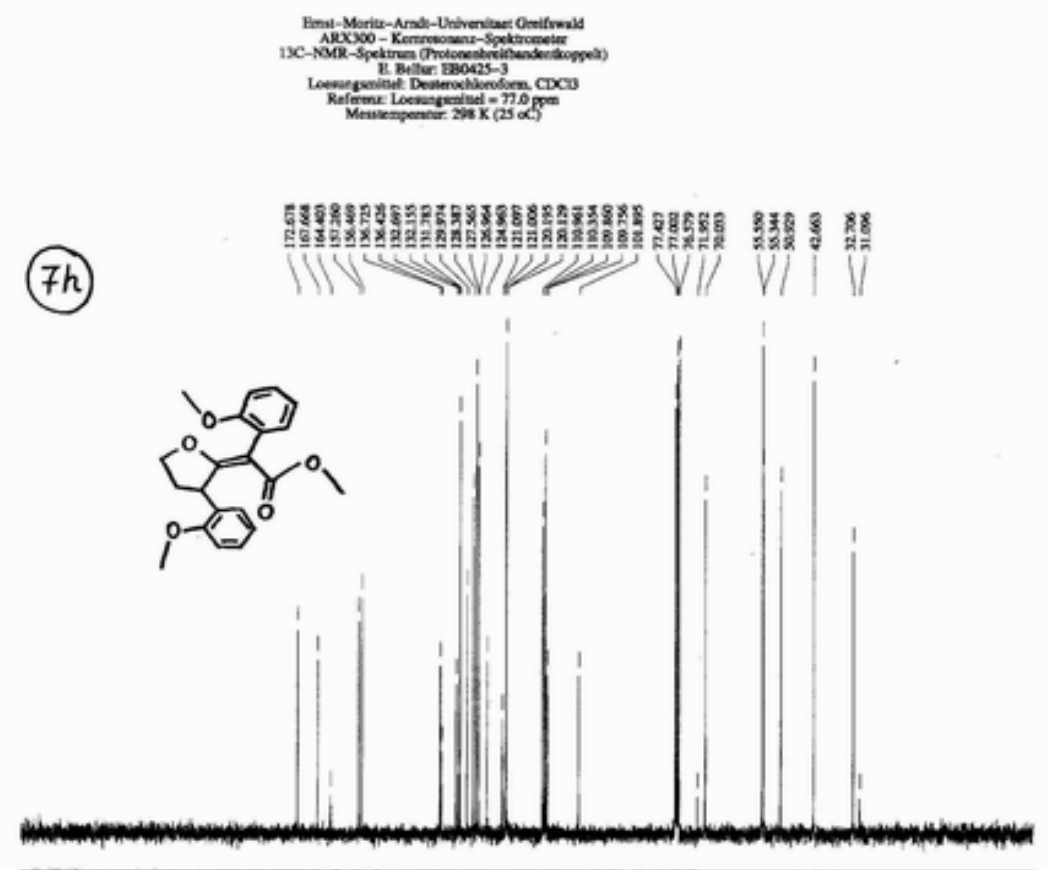

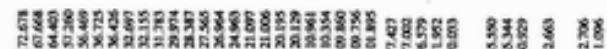

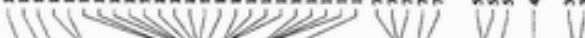

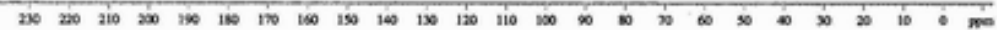
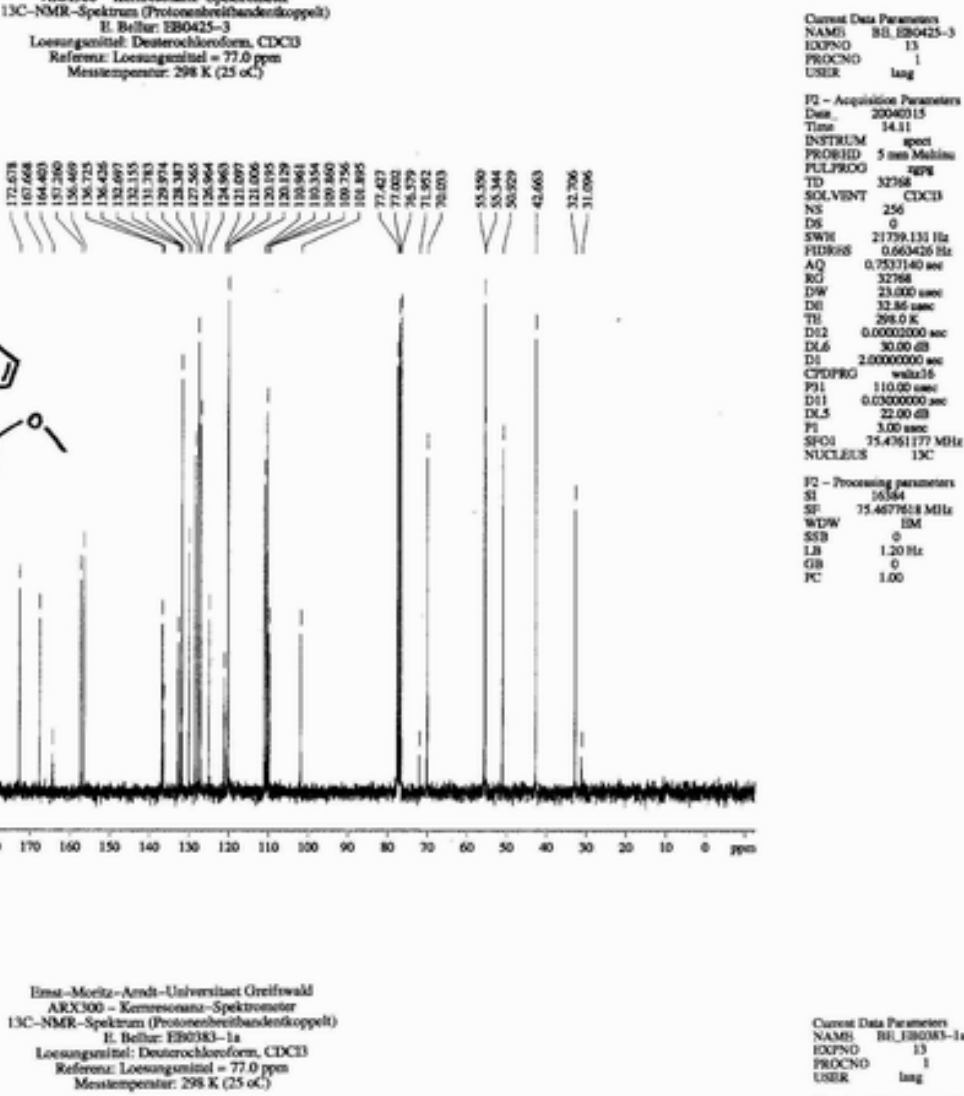

(8a)
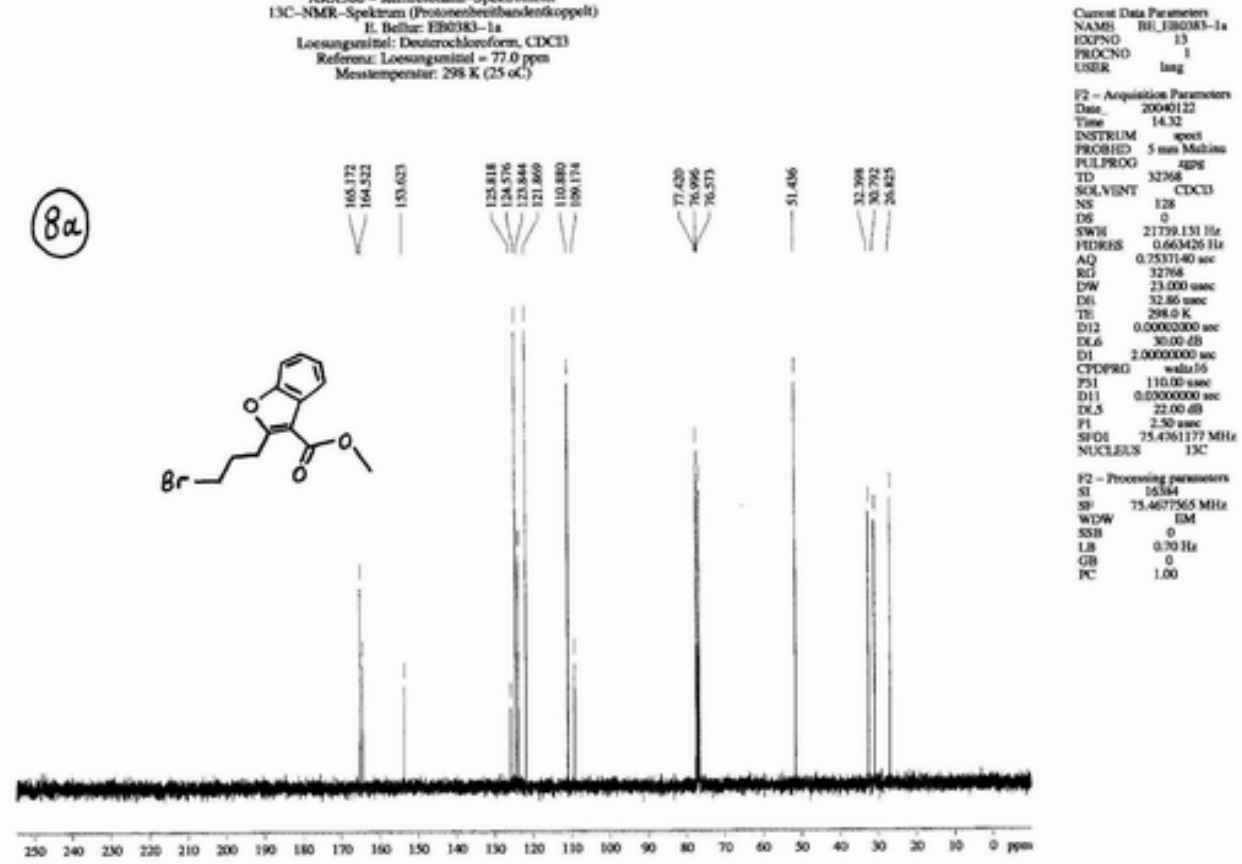
EB0568 - 1

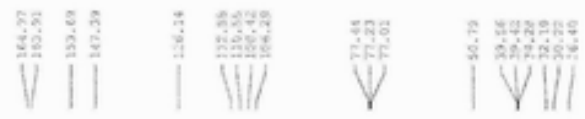

(86)
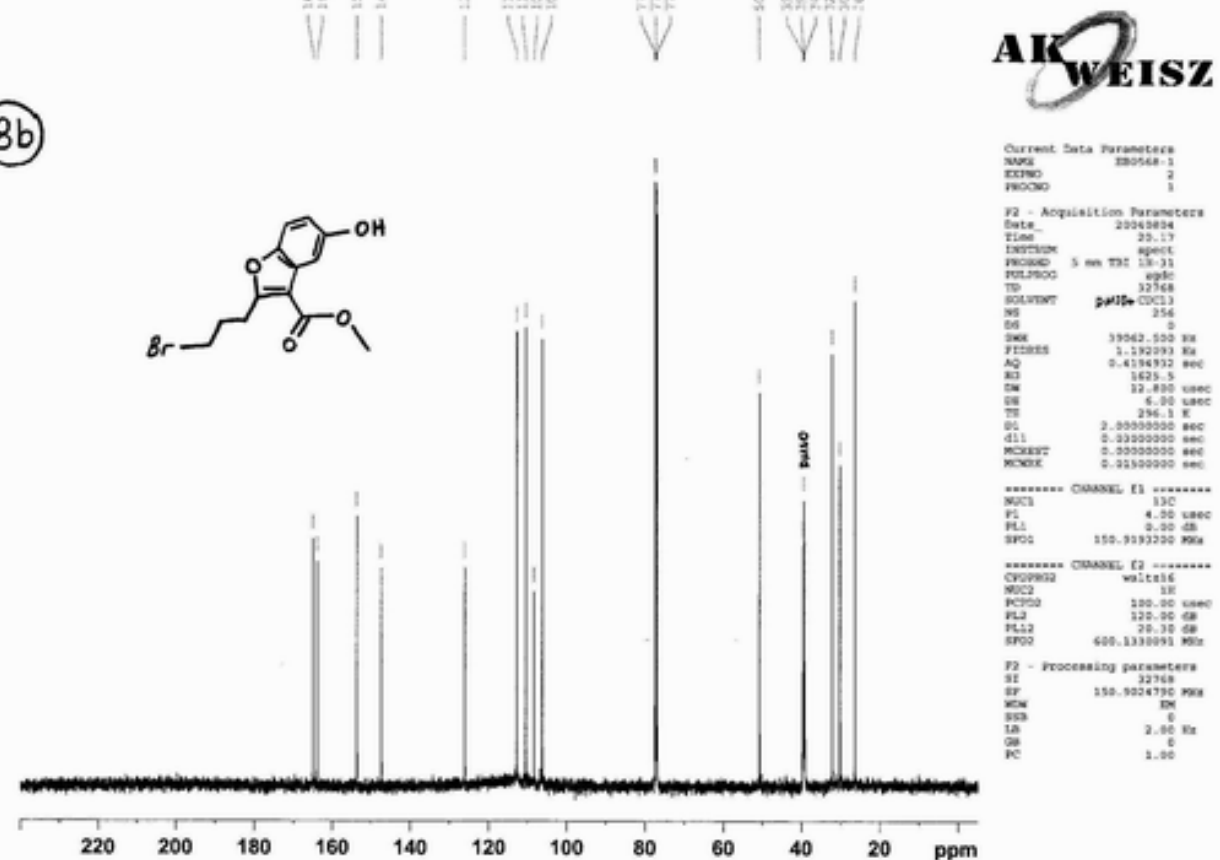

BB0570-1

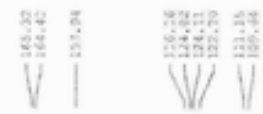

(8c)

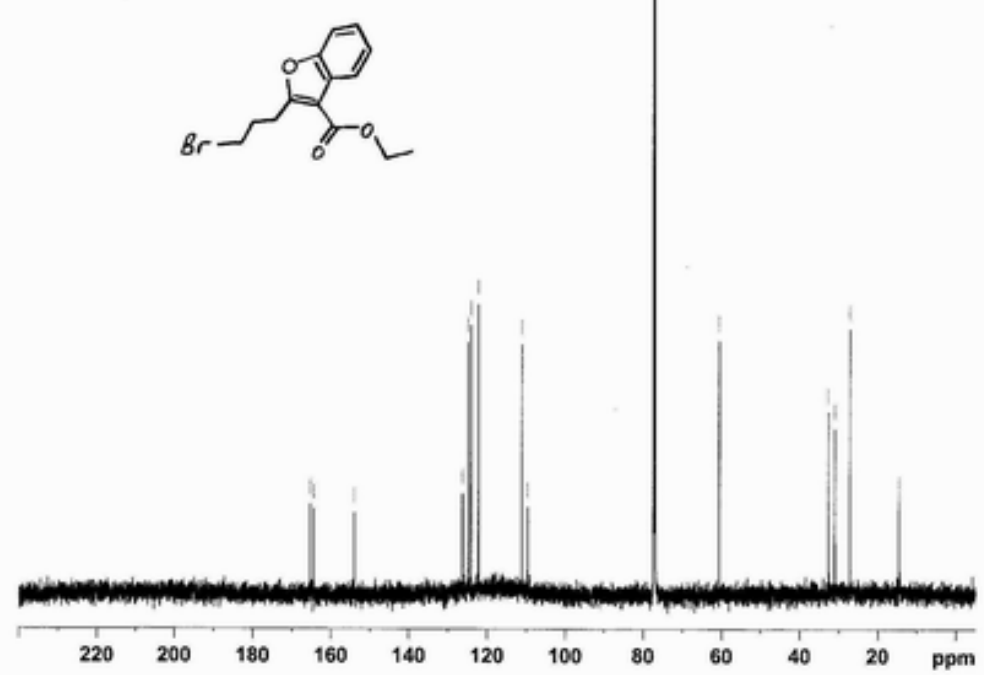

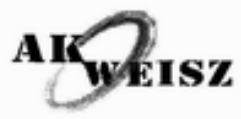

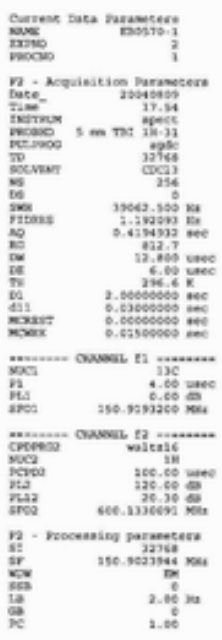




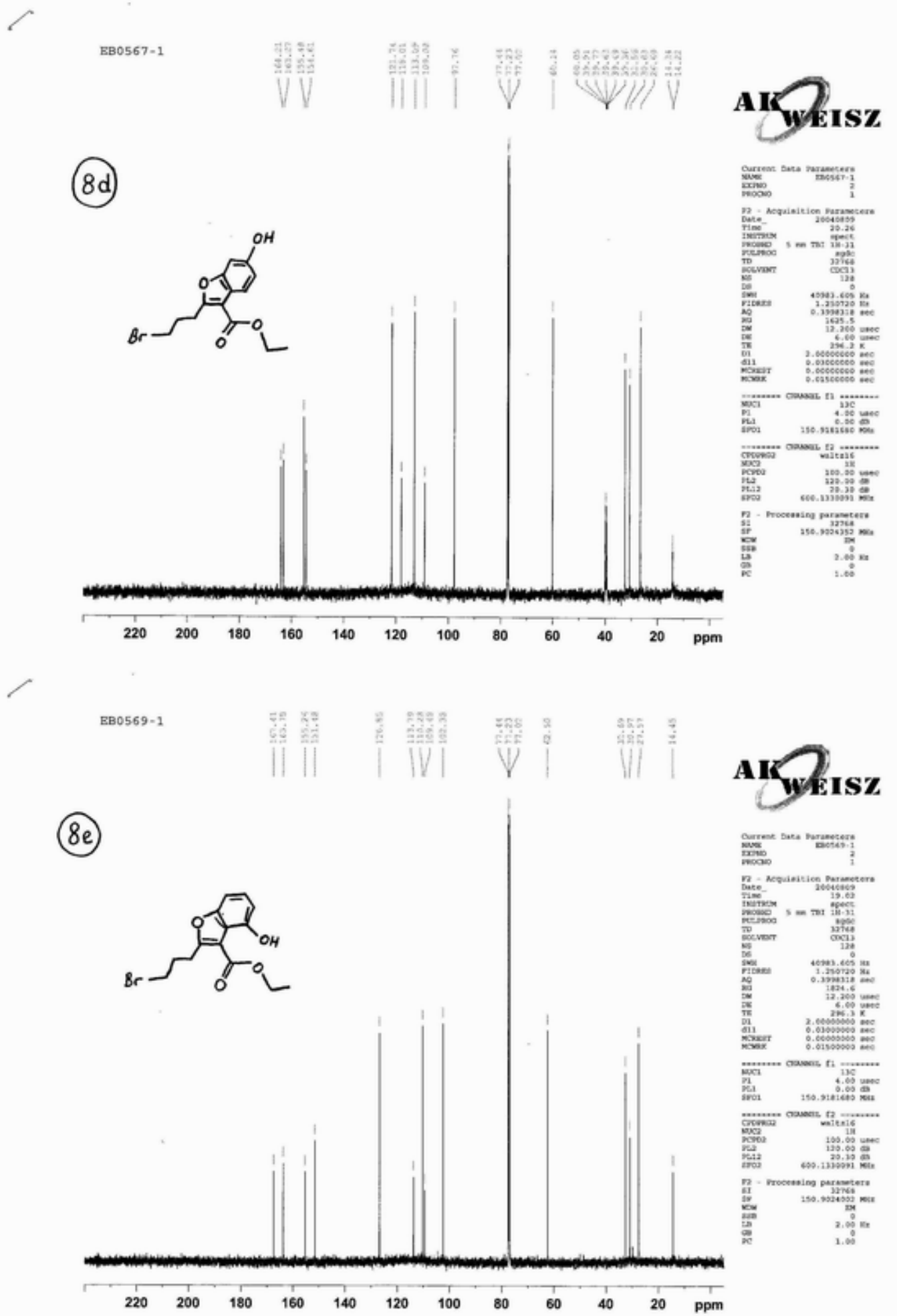




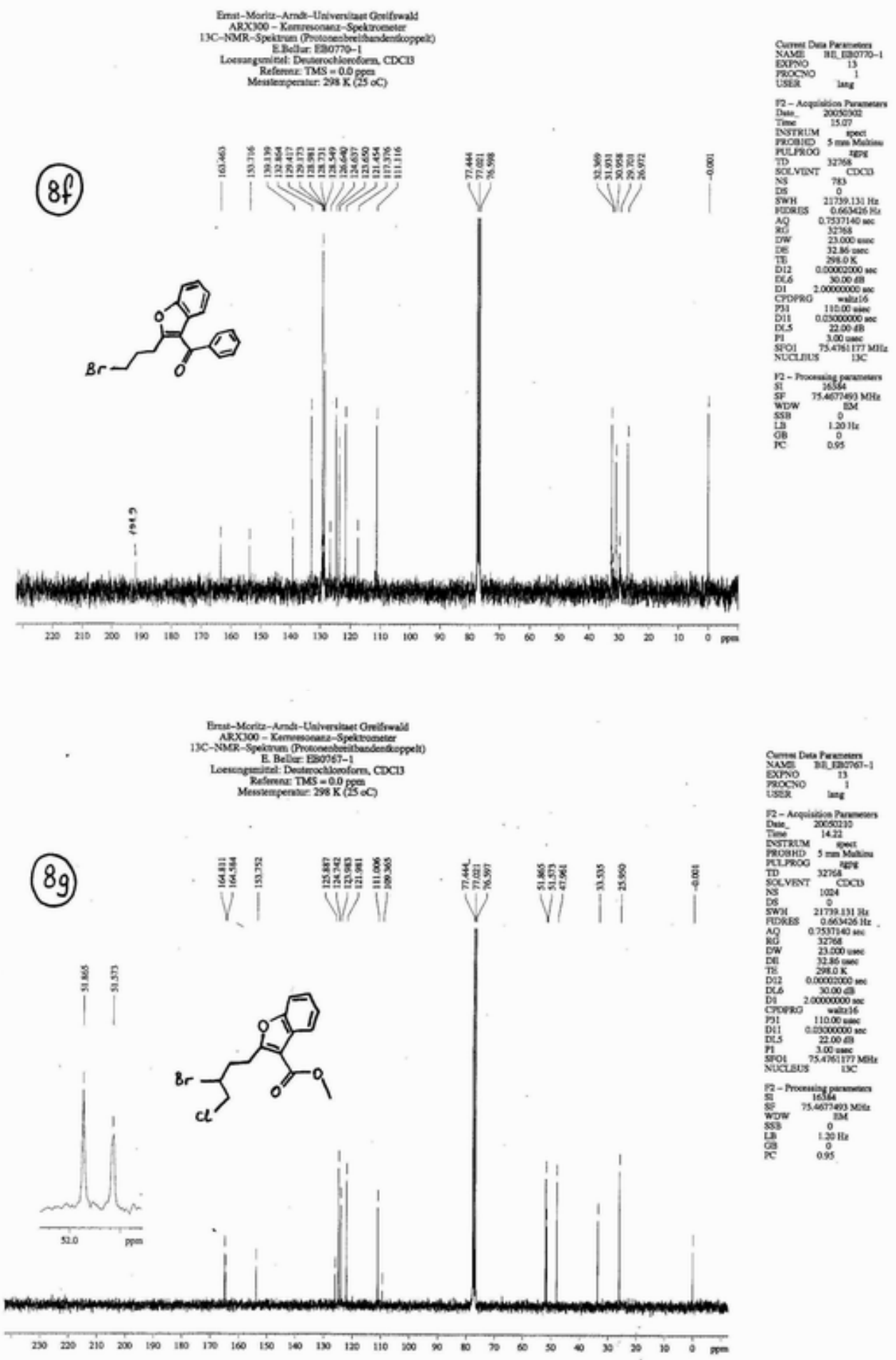




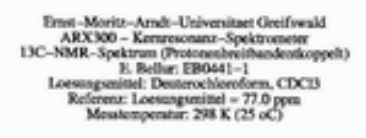

(8h)
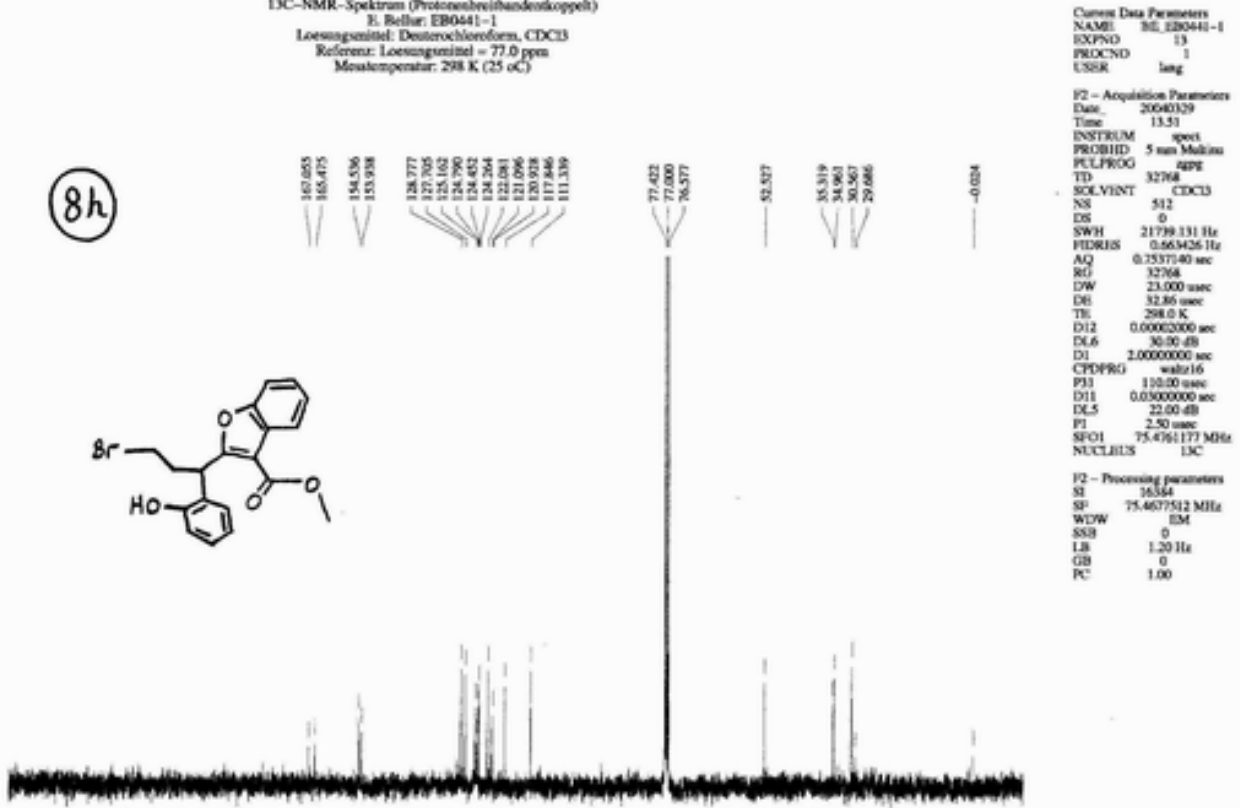

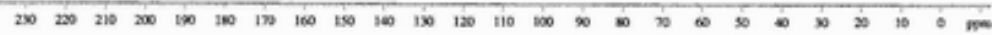

BB 0366-1
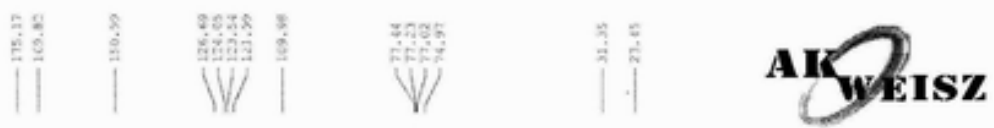

E-9a)
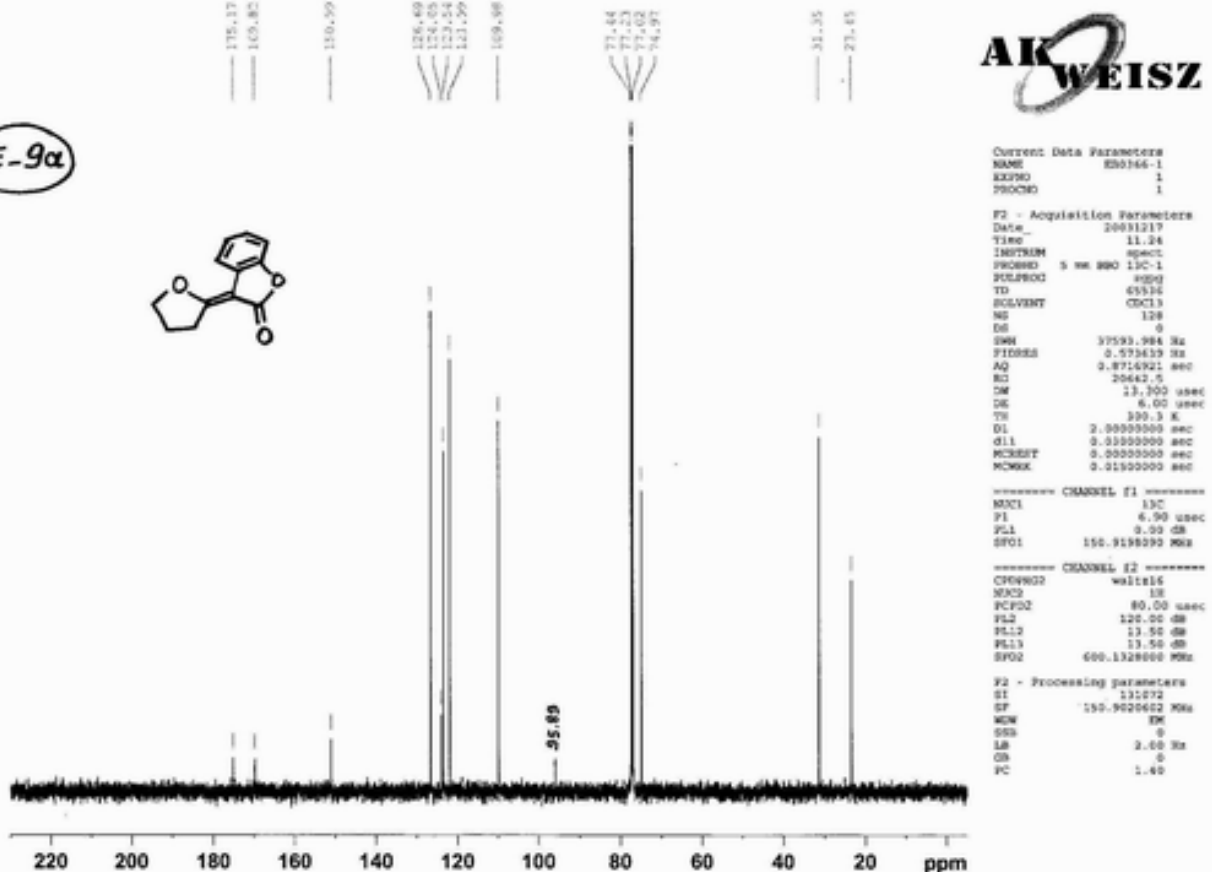
EB $0366-3$

(2-9a.
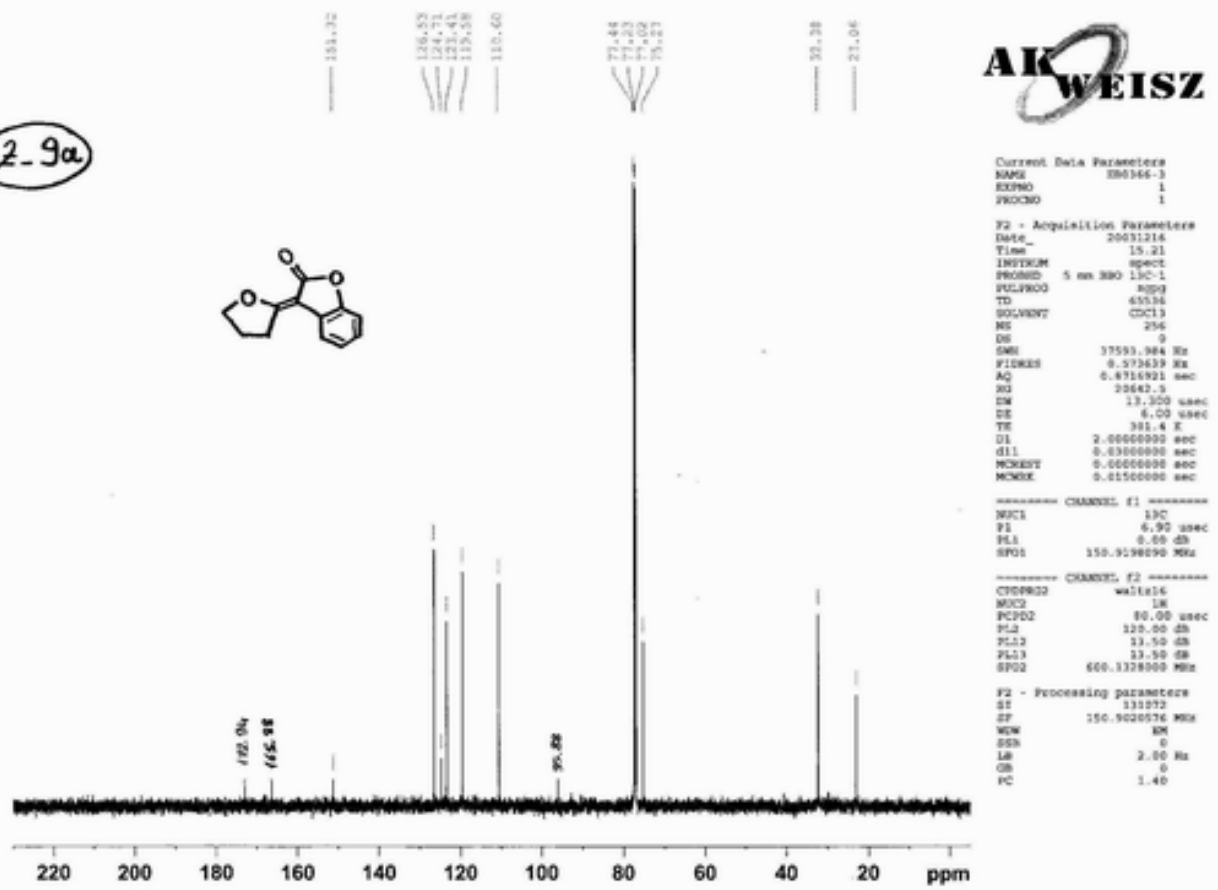

E-9b
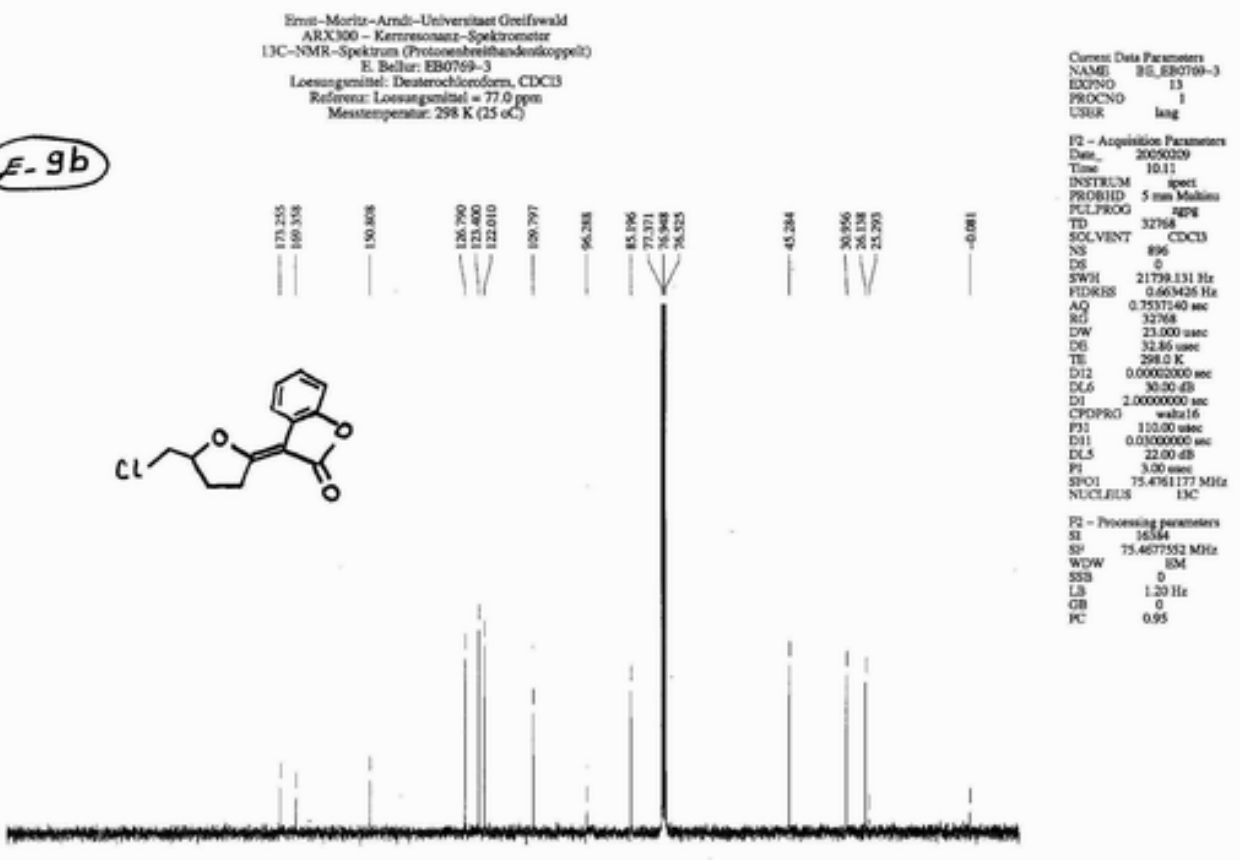

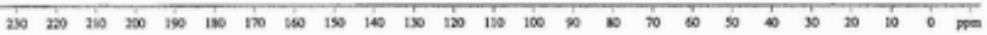




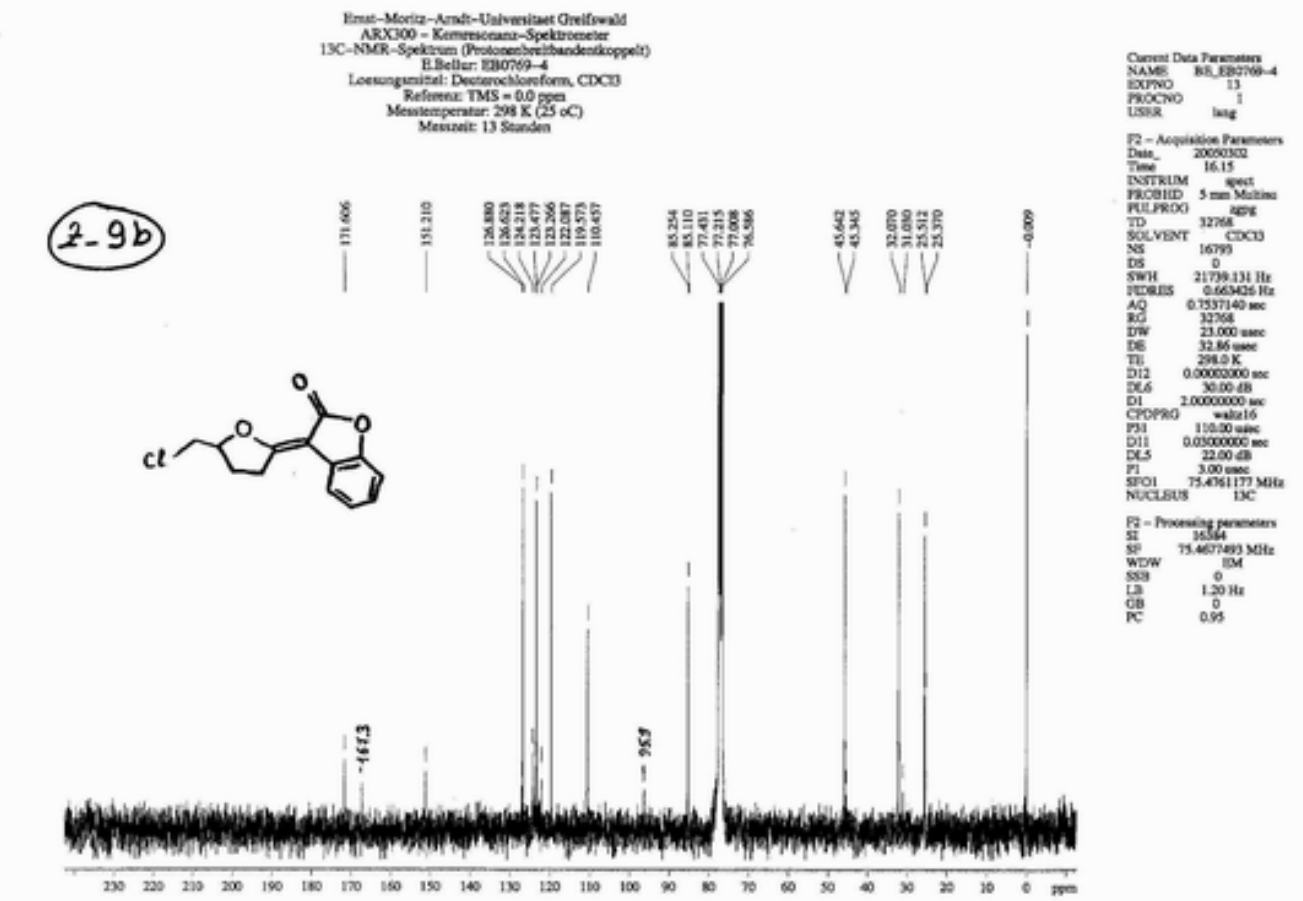

